\title{
Acids Direct 2-Styrylcyclobutanone into Two Distinctly Different Reaction Pathways
}

\author{
Masahiro Murakami,* Sho Kadowaki, Atsushi Fujimoto, Mitsuru Ishibashi, \\ and Takanori Matsuda \\ Department of Synthetic Chemistry and Biological Chemistry, Kyoto University, \\ Katsura, Kyoto 615-8510, Japan
}

\section{Supporting Information}

General. All reactions were carried out under a nitrogen atmosphere. Column chromatography was performed with silica gel $60 \mathrm{~N}$ (Kanto). Preparative thin-layer chromatography was performed with silica gel $60 \mathrm{PF}_{254}$ (Merck). ${ }^{1} \mathrm{H}$ and ${ }^{13} \mathrm{C}$ NMR spectra were recorded on a Varian Gemini $2000\left({ }^{1} \mathrm{H}\right.$ at $300.07 \mathrm{~Hz}$ and ${ }^{13} \mathrm{C}$ at $\left.75.46 \mathrm{~Hz}\right)$ spectrometer or a Varian Mercury $400\left({ }^{1} \mathrm{H}\right.$ at $\left.400.44 \mathrm{MHz}\right)$. All NMR data were obtained in $\mathrm{CDCl}_{3}$. Proton chemical shifts were referenced to the residual proton signal of the solvent at 7.26 ppm. Carbon chemical shifts were referenced to the carbon signal of the solvent at 77.00 ppm. High resolution mass spectra (EI) were recorded on a JEOL JMS-SX102A spectrometer. IR spectra were recorded on a Shimadzu FTIR-8100 spectrometer.

Materials. Unless otherwise noted, all chemicals and anhydrous solvents were obtained from commercial suppliers and used as received. Chlorobenzene and 1,4-dioxane were distilled from $\mathrm{P}_{4} \mathrm{O}_{10}$ and sodium-benzophenone ketyl, respectively, under nitrogen atmosphere. Ytterbium(III) chloride was prepared by heating $\mathrm{YbCl}_{3} \cdot 6 \mathrm{H}_{2} \mathrm{O}$ under reduced pressure $\left(140-150 \quad{ }^{\circ} \mathrm{C} / 5 \mathrm{mmHg}\right)$ for $2 \mathrm{~h}$. Triphenylboroxin-pyridine complex, ${ }^{1}$ cyclopropyldiphenylsulfonium tetrafluoroborate, ${ }^{2}$ and 2-vinylbenzophenone ${ }^{3}$ were prepared according to the literature procedures.

(1) Kerins, F.; O'Shea, D. F. J. Org. Chem. 2002, 67, 4968.

(2) Bogdanowicz, M. J.; Trost, B. M. Org. Synth. Coll. Vol. VI, p 34.

(3) Jason, M. E. Tetrahedron Lett. 1982, 23, 1635. 


\section{Typical Procedures for the Preparation of 2-(o-Styryl)cyclobutanones 1.}

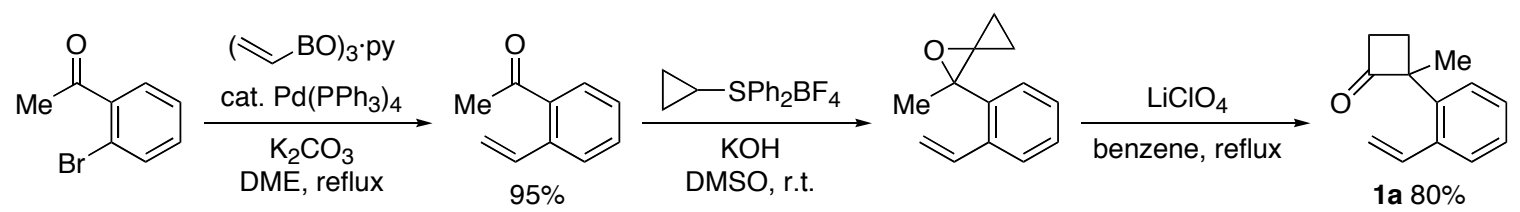

Procedure A: 2-Methyl-2-(2-vinylphenyl)cyclobutanone (1a). A solution of $o$ bromoacetophenone $(8.07 \mathrm{~g}, 40.6 \mathrm{mmol})$ and tetrakis(triphenylphosphine)palladium(0) (463 mg, $0.40 \mathrm{mmol}$ ) in 1,2-dimethoxyethane $(320 \mathrm{~mL}$ ) was stirred for $20 \mathrm{~min}$. To the mixture were added $\mathrm{K}_{2} \mathrm{CO}_{3}(5.61 \mathrm{~g}, \quad 41.0 \mathrm{mmol})$, water $(100 \mathrm{~mL})$, and trivinylboroxin-pyridine complex $(7.23 \mathrm{~g}, 30.0 \mathrm{mmol})$, and the mixture was stirred under reflux $14 \mathrm{~h}$. The reaction mixture was extracted with $\mathrm{Et}_{2} \mathrm{O}$, dried over $\mathrm{Na}_{2} \mathrm{SO}_{4}$, diluted with hexane, filtered, and concentrated. The residue was purified by column chromatography on silica gel (hexane:AcOEt $=10: 1)$ to give $o$-vinylacetophenone $(5.66 \mathrm{~g}, 95 \%)$. To a mixture of cyclopropyldiphenylsulfonium tetrafluoroborate $(13.6 \mathrm{~g}, \quad 43.3 \mathrm{mmol}), \quad o-$ vinylacetophenone $(5.14 \mathrm{~g}, 35.2 \mathrm{mmol})$, and DMSO $(90 \mathrm{~mL})$ was added powdered $\mathrm{KOH}$ (3.95 g, $70.4 \mathrm{mmol}$ ), and the mixture was stirred at ambient temperature for $2 \mathrm{~h}$. The reaction mixture was extracted with hexane, washed with saturated $\mathrm{NaHCO}_{3}$ aqueous solution, dried over $\mathrm{Na}_{2} \mathrm{SO}_{4}$, filtered, and concentrated. To the crude oxaspiropentane were added $\mathrm{LiClO}_{4}(372 \mathrm{mg}, 3.5 \mathrm{mmol})$ and benzene $(250 \mathrm{~mL})$, and the mixture was stirred under reflux overnight. The reaction mixture was cooled to ambient temperature, filtered, and concentrated. The residue was purified by column chromatography on silica gel (hexane:AcOEt $=15: 1)$ to give 1a $(5.26 \mathrm{~g}, 80 \%):{ }^{1} \mathrm{H} \mathrm{NMR}(300 \mathrm{MHz}) \delta 2.29$ (ddd, $J=11.3$, 9.9, $6.5 \mathrm{~Hz}, 1 \mathrm{H}), 2.54(\mathrm{dt}, J=10.9,7.1 \mathrm{~Hz}, 1 \mathrm{H}), 3.06(\mathrm{ddd}, J=18.1,10.7,6.5 \mathrm{~Hz}, 1 \mathrm{H})$, 3.15 (ddd, $J=18.1,9.7,7.1 \mathrm{~Hz}, 1 \mathrm{H}), 5.33(\mathrm{~d}, J=10.8 \mathrm{~Hz}, 1 \mathrm{H}), 5.67(\mathrm{~d}, J=17.2 \mathrm{~Hz}, 1 \mathrm{H})$, $6.99(\mathrm{dd}, J=17.2,10.8 \mathrm{~Hz}, 1 \mathrm{H}), 7.19-7.27(\mathrm{~m}, 2 \mathrm{H}), 7.56-7.60(\mathrm{~m}, 2 \mathrm{H}) ;{ }^{13} \mathrm{C}$ NMR $\delta 25.4$, 27.5, 42.7, 67.6, 115.3, 125.6, 127.03, 127.08, 127.6, 135.0, 135.3, 139.5, 212.2; HRMS (EI) calcd for $\mathrm{C}_{13} \mathrm{H}_{14} \mathrm{O}\left(\mathrm{M}^{+}\right)$186.1045, found 186.1044 . 

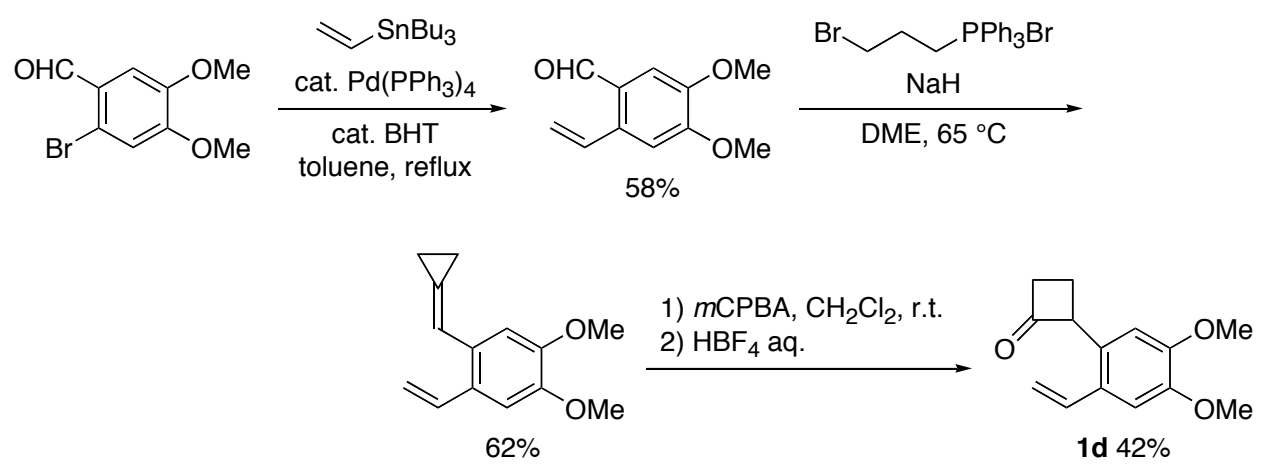

Procedure B: 2-(4,5-dimethoxy-2-vinylphenyl)cyclobutanone (1d). To a mixture of tetrakis(triphenylphosphine)palladium(0) (231 mg, $0.20 \mathrm{mmol}), 2,6$-di-t-butyl-p-cresol (331 $\mathrm{mg}, 1.50 \mathrm{mmol})$, and 2-bromo-4,5-dimethoxybenzaldehyde (2.61 g, $10.7 \mathrm{mmol})$ in toluene $(16.0 \mathrm{~mL})$ was added a toluene solution $(9.0 \mathrm{~mL})$ of tributylvinylstannane $(3.81 \mathrm{~g}, 12.0$ $\mathrm{mmol}$ ), and the mixture was stirred under reflux for $7 \mathrm{~h}$. The reaction mixture was poured into saturated $\mathrm{KF}$ aqueous solution, extracted with $\mathrm{Et}_{2} \mathrm{O}$, dried over $\mathrm{MgSO}_{4}$, and concentrated. The residue was purified by column chromatography on silica gel (hexane:AcOEt $=3: 1)$ to give 2-vinyl-4,5-dimethoxybenzaldehyde $(1.18 \mathrm{~g}, 58 \%)$. A suspension of dry $\mathrm{NaH}(192 \mathrm{mg}, 8.00 \mathrm{mmol})$, 3-bromopropyltriphenylphosphonium bromide (1.86 g, $4.01 \mathrm{mmol})$, and a few drops of EtOH in 1,2-dimethoxyethane $(6.0 \mathrm{~mL})$ was stirred at $65{ }^{\circ} \mathrm{C}$ for $4 \mathrm{~h}$. To the mixture was added a DME solution $(3.0 \mathrm{~mL})$ of 2-vinyl4,5-dimethoxybenzaldehyde ( $769 \mathrm{mg}, 4.00 \mathrm{mmol}$ ), and the mixture was stirred at $65^{\circ} \mathrm{C}$ for $3 \mathrm{~h}$. The solvent was removed under reduced pressure, and the residue was purified by column chromatography on silica gel (hexane:AcOEt $=3: 1$ ) to give 1cyclopropylidenemethyl-4,5-dimethoxy-2-vinylbenzene (533 mg, 62\%). To a $\mathrm{CH}_{2} \mathrm{Cl}_{2}$ solution (14 mL) of 1-cyclopropylidenemethyl-4,5-dimethoxy-2-vinylbenzene (533 mg, $2.46 \mathrm{mmol}$ ) was added $m$-chloroperbenzoic acid $(551 \mathrm{mg}, 2.46 \mathrm{mmol})$ at $0{ }^{\circ} \mathrm{C}$, and the mixture was stirred at $0{ }^{\circ} \mathrm{C}$ for $8 \mathrm{~h}$. The reaction mixture was washed with saturated $\mathrm{NaHCO}_{3}$ aqueous solution and brine, dried over $\mathrm{MgSO}_{4}$, and concentrated. The residue was dissolved in $\mathrm{CH}_{2} \mathrm{Cl}_{2}(21 \mathrm{~mL})$, and $15 \% \mathrm{HBF}_{4}$ aqueous solution was added to the solution at ambient temperature. After being stirred for $9 \mathrm{~h}$, the mixture was extracted with $\mathrm{CH}_{2} \mathrm{Cl}_{2}$, washed with saturated $\mathrm{NaHCO}_{3}$ aqueous solution and brine, dried over $\mathrm{MgSO}_{4}$, and concentrated. The residue was purified by preparative thin-layer chromatography 
(hexane:AcOEt = 3/1) to give 1d $(243 \mathrm{mg}, 42 \%):{ }^{1} \mathrm{H}$ NMR $(300 \mathrm{MHz}) \delta 2.11$ (ddt, $J=10.6$, 9.7, 8.2 Hz, 1H), 2.57 (dq, $J=4.7,10.8 \mathrm{~Hz}, 1 \mathrm{H}$ ), 3.03 (dddd, $J=18.0,9.7,4.7,2.3 \mathrm{~Hz}, 1 \mathrm{H}$ ), 3.22 (dddd, $J=18.0,10.6,8.2,2.3 \mathrm{~Hz}, 1 \mathrm{H}), 3.88$ (s, 3H), 3.90 (s, 3H), 4.73 (ddt, $J=10.6$, 8.2, $2.3 \mathrm{~Hz}, 1 \mathrm{H}), 5.25(\mathrm{dd}, J=10.8,1.2 \mathrm{~Hz}, 1 \mathrm{H}), 5.55(\mathrm{dd}, J=17.1,1.2 \mathrm{~Hz}, 1 \mathrm{H}), 6.83(\mathrm{dd}$, $J=17.1,10.8 \mathrm{~Hz}, 1 \mathrm{H}), 6.85(\mathrm{~s}, 1 \mathrm{H}), 7.02(\mathrm{~s}, 1 \mathrm{H}) ;{ }^{13} \mathrm{C} \mathrm{NMR} \delta$ 19.4, 44.6, 55.9, 56.0, 61.7, 108.9, 109.6, 114.4, 127.0, 128.8, 133.9, 148.0, 148.7, 208.4; HRMS calcd for $\mathrm{C}_{14} \mathrm{H}_{16} \mathrm{O}_{3}$ $\left(\mathrm{M}^{+}\right)$232.1099, found 232.1095.

Procedure for the Synthesis of Naphthalenes 2.

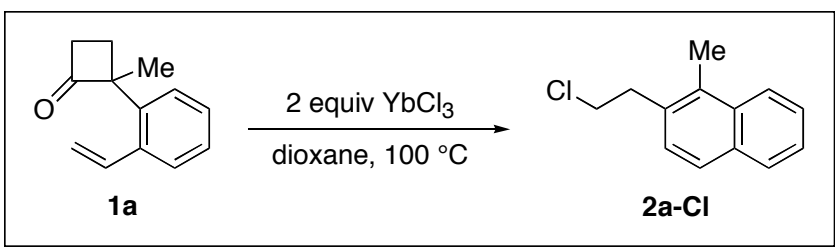

2-(2-Chloroethyl)-1-methylnaphthalene (2a-Cl). To a suspension of $\mathrm{YbCl}_{3}(224 \mathrm{mg}, 0.80$ mmol $)$ in 1,4-dioxane $(2.0 \mathrm{~mL})$ was added a 1,4-dioxane solution $(6.0 \mathrm{~mL})$ of $2-(o$ styryl)cyclobutanone 1a $(74.5 \mathrm{mg}, 0.40 \mathrm{mmol})$. After heating under reflux for $24 \mathrm{~h}$, the mixture was passed through a plug of Florisil ${ }^{\circledR}$ eluting with AcOEt. The solvent was removed under reduced pressure, and the residue was purified by preparative thin-layer chromatography of silica gel (hexane:AcOEt $=10: 1)$ to give $\mathbf{2 a}(67.7 \mathrm{mg}, 83 \%):{ }^{1} \mathrm{H} \mathrm{NMR}$ $(400 \mathrm{MHz}) \delta 2.68(\mathrm{~s}, 3 \mathrm{H}), 3.32(\mathrm{~d}, J=7.8 \mathrm{~Hz}, 2 \mathrm{H}), 3.73(\mathrm{~d}, J=7.8 \mathrm{~Hz}, 2 \mathrm{H}), 7.31(\mathrm{~d}, J=$ $8.4 \mathrm{~Hz}, 1 \mathrm{H}), 7.45-7.56(\mathrm{~m}, 2 \mathrm{H}), 7.69(\mathrm{~d}, J=8.4 \mathrm{~Hz}, 1 \mathrm{H}), 7.82(\mathrm{dd}, J=8.2,1.0 \mathrm{~Hz}, 1 \mathrm{H})$, $8.06(\mathrm{dd}, J=8.4,0.8 \mathrm{~Hz}, 1 \mathrm{H}) ;{ }^{13} \mathrm{C} \mathrm{NMR} \delta 14.3,37.7,44.0,124.1,125.3,126.1,126.4$, 128.2, 128.5, 131.8, 132.7, 133.0, 133.1; HRMS calcd for $\mathrm{C}_{13} \mathrm{H}_{13} \mathrm{Cl}\left(\mathrm{M}^{+}\right)$204.0706, found 204.0704 .

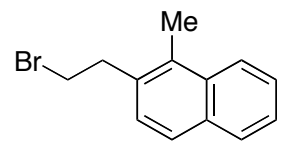


2-(2-Bromoethyl)-1-methylnaphthalene (2a-Br). ${ }^{4}$ A mixture of $\mathbf{1 a}(55.9 \mathrm{mg}, 0.30 \mathrm{mmol})$ and $\mathrm{YbBr}_{3}(248 \mathrm{mg}, 0.60 \mathrm{mmol})$ in 1,4-dioxane $(6.0 \mathrm{~mL})$ was heated under reflux for $5 \mathrm{~h}$. The reaction mixture was passed through a plug of Florisil ${ }^{\circledR}$ (AcOEt). Purification by preparative thin-layer chromatography of silica gel (hexane:AcOEt $=10: 1$ ) afforded 2a-Br (64.3 mg, 86\%): ${ }^{1} \mathrm{H}$ NMR (300 MHz) $\delta 2.68(\mathrm{~s}, 3 \mathrm{H}), 3.40(\mathrm{t}, J=8.0 \mathrm{~Hz}, 2 \mathrm{H}), 3.58(\mathrm{t}, J=$ $8.0 \mathrm{~Hz}, 2 \mathrm{H}), 7.31(\mathrm{~d}, J=8.4 \mathrm{~Hz}, 2 \mathrm{H}), 7.46-7.58(\mathrm{~m}, 2 \mathrm{H}), 7.70(\mathrm{~d}, J=8.4 \mathrm{~Hz}, 2 \mathrm{H}), 7.83(\mathrm{~d}$, $J=8.1 \mathrm{~Hz}, 2 \mathrm{H}), 8.07(\mathrm{~d}, J=8.4 \mathrm{~Hz}, 2 \mathrm{H}) ;{ }^{13} \mathrm{C}$ NMR $\delta 14.4,31.8,38.0,124.0,125.2,126.0$, 126.4, 128.0, 128.4, 131.6, 132.6, 132.9, 133.9; HRMS calcd for $\mathrm{C}_{13} \mathrm{H}_{13} \mathrm{Br}\left(\mathrm{M}^{+}\right)$248.0201, found 248.0198 .

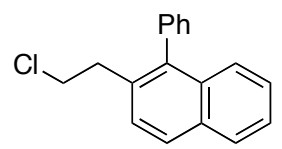

2-(2-Chloroethyl)-1-phenylnaphthalene (2b-Cl). A mixture of $\mathbf{1 b}$ (99.3 mg, $0.40 \mathrm{mmol}$ ) and $\mathrm{YbCl}_{3}(224 \mathrm{mg}, 0.80 \mathrm{mmol})$ in 1,4-dioxane $(8.0 \mathrm{~mL})$ was heated under reflux for $24 \mathrm{~h}$. The reaction mixture was passed through a plug of Florisil ${ }^{\circledR}$ (AcOEt). Purification by preparative thin-layer chromatography of silica gel (hexane:AcOEt $=5: 1$ ) afforded $\mathbf{2 b - C l}$ (84.5 mg, 79\%): mp 59-60 ${ }^{\circ} \mathrm{C} ;{ }^{1} \mathrm{H}$ NMR $(300 \mathrm{MHz}) \delta 3.05(\mathrm{t}, J=7.7 \mathrm{~Hz}, 2 \mathrm{H}), 3.61(\mathrm{t}, J=$ $7.7 \mathrm{~Hz}, 2 \mathrm{H}), 7.26-7.42(\mathrm{~m}, 4 \mathrm{H}), 7.42-7.58(\mathrm{~m}, 5 \mathrm{H}), 7.88(\mathrm{~d}, J=8.4 \mathrm{~Hz}, 2 \mathrm{H}) ;{ }^{13} \mathrm{C} \mathrm{NMR} \delta$ 37.1, 44.4, 125.4, 126.0, 126.5, 127.40, 127.43, 127.69, 127.72, 128.4, 130.1, 132.3, 132.96, 133.01, 138.6, 139.0; HRMS calcd for $\mathrm{C}_{18} \mathrm{H}_{15} \mathrm{Cl}\left(\mathrm{M}^{+}\right)$266.0862, found 266.0866.

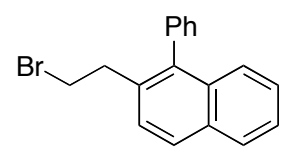

2-(2-Bromoethyl)-1-phenylnaphthalene (2b-Br). A mixture of $\mathbf{1 b}$ (74.5 $\mathrm{mg}, 0.30 \mathrm{mmol})$ and $\mathrm{YbBr}_{3}(248 \mathrm{mg}, 0.60 \mathrm{mmol})$ in 1,4-dioxane $(6.0 \mathrm{~mL})$ was heated under reflux for $5 \mathrm{~h}$. The reaction mixture was passed through a plug of Florisil ${ }^{\circledR}$ (AcOEt). Purification by preparative thin-layer chromatography of silica gel (hexane:AcOEt $=10: 1$ ) afforded $\mathbf{2 b}-\mathbf{B r}$ (87.5 mg, 94\%): $\mathrm{mp} 71-72{ }^{\circ} \mathrm{C} ;{ }^{1} \mathrm{H}$ NMR $\delta 3.13(\mathrm{t}, J=7.8 \mathrm{~Hz}, 2 \mathrm{H}), 3.45(\mathrm{t}, J=7.8 \mathrm{~Hz}, 2 \mathrm{H})$,

(4) $[107517-26-0]$. 
7.28-7.41 (m, 4H), 7.41-7.56 (m, 5H), $7.87(\mathrm{~d}, J=8.1 \mathrm{~Hz}, 2 \mathrm{H}) ;{ }^{13} \mathrm{C}$ NMR $\delta 32.2,37.3$, $125.4,126.0,126.5,127.3,127.4,127.7,127.8,128.4,130.1,132.3,133.0,133.8,138.6$, 138.9.0; HRMS calcd for $\mathrm{C}_{18} \mathrm{H}_{15} \mathrm{Br}\left(\mathrm{M}^{+}\right)$310.0357, found 310.0360 .

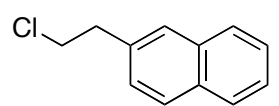

2-(2-Chloroethyl)naphthalene (2c-Cl). ${ }^{5}$ A mixture of $\mathbf{1 c}(87.4 \mathrm{mg}, 0.51 \mathrm{mmol})$ and $\mathrm{YbCl}_{3}$ (280 mg, $1.00 \mathrm{mmol})$ in 1,4-dioxane $(10 \mathrm{~mL})$ was heated under reflux for $32 \mathrm{~h}$. The reaction mixture was passed through a plug of Florisil ${ }^{\circledR}\left(\mathrm{CHCl}_{3}\right)$. Purification by preparative thin-layer chromatography of silica gel (hexane:AcOEt = 10:1) afforded 2c-Cl $(66.2 \mathrm{mg}$, 68\%). ${ }^{1} \mathrm{H}$ NMR (400 MHz) $\delta 3.26(\mathrm{~d}, J=7.3 \mathrm{~Hz}, 2 \mathrm{H}), 3.83(\mathrm{~d}, J=7.3 \mathrm{~Hz}, 2 \mathrm{H}), 7.37$ (dd, $J$ $=8.4,1.6 \mathrm{~Hz}, 1 \mathrm{H}), 7.46-7.54(\mathrm{~m}, 2 \mathrm{H}), 7.70(\mathrm{~s}, 1 \mathrm{H}), 7.81-7.88(\mathrm{~m}, 4 \mathrm{H}) ;{ }^{13} \mathrm{C} \mathrm{NMR} \delta 39.2$, $44.8,125.7,126.2,127.0,127.4,127.6,127.7,128.3$, 132.4, 133.5, 135.5; HRMS calcd for $\mathrm{C}_{12} \mathrm{H}_{11} \mathrm{Cl}\left(\mathrm{M}^{+}\right)$190.0549, found 190.0551.

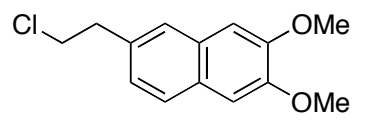

6-(2-Chloroethyl)-2,3-dimethoxynaphthalene (2d-Cl). ${ }^{6}$ A mixture of 1d (69.7 mg, 0.30 $\mathrm{mmol})$ and $\mathrm{YbCl}_{3}(168 \mathrm{mg}, 0.60 \mathrm{mmol})$ in 1,4-dioxane $(6.0 \mathrm{~mL})$ was heated under reflux for $2 \mathrm{~h}$. The reaction mixture was passed through a plug of Florisil ${ }^{\circledR}$ (AcOEt). Purification by preparative thin-layer chromatography of silica gel (hexane:AcOEt $=5: 2$ ) afforded $2 \mathbf{d}$ Cl $(48.7 \mathrm{mg}, 65 \%) .{ }^{1} \mathrm{H} \mathrm{NMR}(300 \mathrm{MHz}) \delta 3.19(\mathrm{t}, J=7.5 \mathrm{~Hz}, 2 \mathrm{H}), 3.79(\mathrm{t}, J=7.5 \mathrm{~Hz}, 2 \mathrm{H})$, 3.99 (s, 3H), 4.00 (s, 3H), 7.10 (d, $J=4.8 \mathrm{~Hz}, 2 \mathrm{H}), 7.20(\mathrm{dd}, J=8.3,1.7 \mathrm{~Hz}, 1 \mathrm{H}), 7.53$ (s, $1 \mathrm{H}), 7.65(\mathrm{~d}, J=8.3 \mathrm{~Hz}, 1 \mathrm{H}) ;{ }^{13} \mathrm{C} \mathrm{NMR} \delta 39.3,45.1,55.8,106.0,106.1,125.2,125.9$, 126.6, 128.0, 129.2, 133.8, 149.3, 149.6; HRMS calcd for $\mathrm{C}_{14} \mathrm{H}_{15} \mathrm{ClO}_{2}\left(\mathrm{M}^{+}\right)$250.0761, found 250.0760 .

(5) [20849-71-2].

(6) [72337-08-7]. 


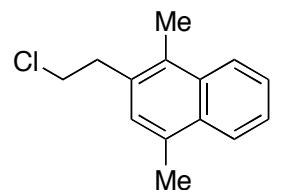

2-(2-Chloroethyl)-1,4-dimethylnaphthalene (2e-Cl). A mixture of $\mathbf{1 e}(108.9 \mathrm{mg}, 0.54$ $\mathrm{mmol})$ and $\mathrm{YbCl}_{3}(304 \mathrm{mg}, 1.09 \mathrm{mmol})$ in 1,4-dioxane $(2.7 \mathrm{~mL})$ was heated under reflux for $2 \mathrm{~h}$. The reaction mixture was passed through a plug of Florisil ${ }^{\circledR}$ (AcOEt). Purification by preparative thin-layer chromatography of silica gel (hexane:AcOEt $=10: 1)$ afforded 2eCl (60.5 mg, 51\%). ${ }^{1} \mathrm{H}$ NMR (300 MHz) $\delta 2.64(\mathrm{~s}, 3 \mathrm{H}), 2.66(\mathrm{~s}, 3 \mathrm{H}), 3.22-3.29(\mathrm{~m}, 2 \mathrm{H})$, 3.66-3.73 (m, 2H), 7.14 (s, 1H), 7.46-7.56 (m, 2H), 7.94-8.00 (m, 1H), 8.02-8.09 (m, 1H); ${ }^{13} \mathrm{C}$ NMR $\delta 14.4,19.3,37.8,44.1,124.5,124.6,125.1,125.7,129.0,129.7,131.8,132.3$, 132.5, 133.0; HRMS calcd for $\mathrm{C}_{14} \mathrm{H}_{15} \mathrm{Cl} 218.0862$, found 218.0865.<smiles>Cc1c(CCCl)c2ccccc2c2ccccc12</smiles>

9-(2-Chloroethyl)-10-methylphenanthrene (2f-Cl). A mixture of $\mathbf{1 f}(94.5 \mathrm{mg}, 0.40 \mathrm{mmol})$ and $\mathrm{YbCl}_{3}(224 \mathrm{mg}, 0.80 \mathrm{mmol})$ in $\mathrm{Bu}_{2} \mathrm{O}(8.0 \mathrm{~mL})$ was heated under reflux for $2 \mathrm{~h}$. The reaction mixture was passed through a plug of Florisil $^{\circledR}$ (AcOEt). Purification by preparative thin-layer chromatography of silica gel (hexane:AcOEt $=10: 1)$ afforded $\mathbf{2 f - C l}$ (49.7 mg, 49\%): ${ }^{1} \mathrm{H}$ NMR (300 MHz) $\delta 2.77$ (s, 3H), 3.68-3.81 (m, 4H), 7.61-7.69 (m, 4H), 8.08-8.16 (m, 2H), 8.70-8.75 (m, 2H); ${ }^{13} \mathrm{C}$ NMR $\delta 15.9,33.1,42.8,122.7,123.1,123.8$, 124.8, 125.7, 126.2, 126.8, 127.0, 129.1, 129.75, 129.78, 130.7, 131.0, 131.7; HRMS calcd for $\mathrm{C}_{17} \mathrm{H}_{15} \mathrm{Cl}\left(\mathrm{M}^{+}\right)$254.0862, found 254.0862.

Procedure for the Synthesis of Eight-Membered Ring Ketones 5.

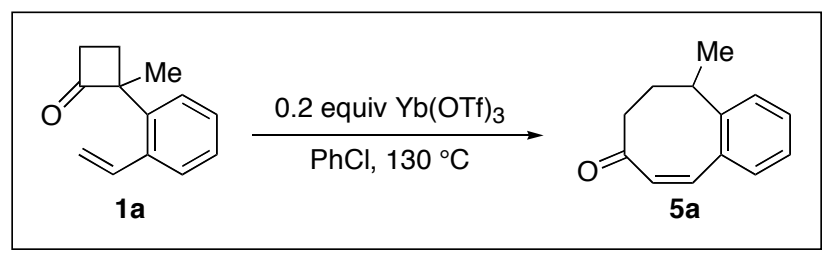


10-Methyl-9,10-dihydrobenzocycloocten-7(8H)-one (5a). To a suspension of $\mathrm{Yb}(\mathrm{OTf})_{3}$ (49.6 mg, $0.080 \mathrm{mmol})$ in chlorobenzene $(2.0 \mathrm{~mL})$ was added a chlorobenzene solution (6.0 $\mathrm{mL})$ of 2-(o-styryl)cyclobutanone $1 \mathrm{a}(74.5 \mathrm{mg}, 0.40 \mathrm{mmol})$. After heating under reflux for $3 \mathrm{~h}$, the mixture was passed through a pad of Florisil ${ }^{\circledR}$ (AcOEt). The solvent was removed under reduced pressure, and the residue was purified by preparative thin-layer chromatography of silica gel (toluene:AcOEt = 15:1) to give 5a $(61.4 \mathrm{mg}, 82 \%)$ : IR (nujol) $1653.2 \mathrm{~cm}^{-1} ;{ }^{1} \mathrm{H}$ NMR (400 MHz) $\delta 1.34(\mathrm{~d}, J=6.8 \mathrm{~Hz}, 3 \mathrm{H}), 1.56-1.66(\mathrm{~m}, 1 \mathrm{H}), 2.14-2.24$ $(\mathrm{m}, 2 \mathrm{H}), 2.34-2.44(\mathrm{~m}, 1 \mathrm{H}), 3.11-3.22(\mathrm{~m}, 1 \mathrm{H}), 6.19(\mathrm{dd}, J=12.7,1.8 \mathrm{~Hz}, 1 \mathrm{H}), 7.12(\mathrm{~d}, J=$ $12.7 \mathrm{~Hz}, 1 \mathrm{H}), 7.25-7.32(\mathrm{~m}, 2 \mathrm{H}), 7.37-7.42(\mathrm{~m}, 2 \mathrm{H}) ;{ }^{13} \mathrm{C} \mathrm{NMR} \delta$ 19.6, 33.5, 39.0, 40.8, $125.1,126.1,129.7,130.2,132.3,135.7,141.2,144.6,203.9$; HRMS calcd for $\mathrm{C}_{13} \mathrm{H}_{14} \mathrm{O}$ $\left(\mathrm{M}^{+}\right)$186.1045, found 186.1046.

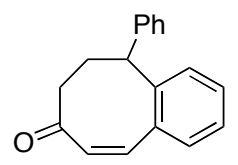

10-Phenyl-9,10-dihydrobenzocycloocten-7(8H)-one (5b). A mixture of $\mathbf{1 b}(99.3 \mathrm{mg}, 0.40$ $\mathrm{mmol})$ and $\mathrm{Yb}(\mathrm{OTf})_{3}(49.6 \mathrm{mg}, 0.080 \mathrm{mmol})$ in $\mathrm{PhCl}(8.0 \mathrm{~mL})$ was heated under reflux for 10 h. The reaction mixture was passed through a plug of Florisil ${ }^{\circledR}$ (AcOEt). Purification by preparative thin-layer chromatography of silica gel (hexane:AcOEt $=5: 1)$ afforded $\mathbf{5 b}(89.6$ mg, 90\%): mp 94-95 ${ }^{\circ} \mathrm{C} ;{ }^{1} \mathrm{H}$ NMR (300 MHz) $\delta 2.30-2.61(\mathrm{~m}, 4 \mathrm{H}), 4.47(\mathrm{dd}, J=13.4,3.6$ $\mathrm{Hz}, 1 \mathrm{H}), 6.30(\mathrm{dt}, J=12.8,1.6 \mathrm{~Hz}, 1 \mathrm{H}), 6.89$ (d, J= 7.6 Hz, 1H), 7.20-7.34 (m, 9H); ${ }^{13} \mathrm{C}$ NMR $\delta 36.1,38.5,44.1,126.1,126.6,128.27,128.34,129.6,129.8,132.7,135.6,141.1$, 142.9, 144.2, 203.2; HRMS calcd for $\mathrm{C}_{18} \mathrm{H}_{16} \mathrm{O}\left(\mathrm{M}^{+}\right)$248.1201, found 248.1195.

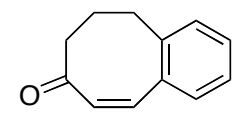

9,10-dihydrobenzocycloocten-7(8H)-one $(5 \mathrm{c}) .{ }^{7}$ A mixture of $\mathbf{1 c}(86.3 \mathrm{mg}, 0.50 \mathrm{mmol})$ and $\mathrm{Yb}(\mathrm{OTf})_{3}(62.0 \mathrm{mg}, 0.10 \mathrm{mmol})$ in $\mathrm{PhCl}(10 \mathrm{~mL})$ was heated under reflux for $8 \mathrm{~h}$. The

(7) [144744-96-7]. Matsuda, T.; Fujimoto, A.; Ishibashi, M.; Murakami, M. Chem. Lett. 2004, 33, 876. 
reaction mixture was passed through a plug of Florisil ${ }^{\circledR}\left(\mathrm{CHCl}_{3}\right)$. Purification by preparative thin-layer chromatography of silica gel (hexane:AcOEt $=10: 1)$ afforded 5c $(54.9 \mathrm{mg}$, 64\%): ${ }^{1} \mathrm{H}$ NMR (400 MHz) $\delta$ 2.04-2.12 (m, 2H), 2.33-2.37 (m, 2H), 2.75-2.80 (m, 2H), $6.16(\mathrm{~d}, J=12.8 \mathrm{~Hz}, 1 \mathrm{H}), 7.11(\mathrm{~d}, J=12.8 \mathrm{~Hz}, 1 \mathrm{H}), 7.25-7.37(\mathrm{~m}, 4 \mathrm{H}) ;{ }^{13} \mathrm{C} \mathrm{NMR} \delta 32.0$, 32.5, 37.9, 126.5, 129.7, 129.9, 130.8, 132.0, 135.7, 140.8, 141.2, 204.3. Anal. Calcd. For $\mathrm{C}_{12} \mathrm{H}_{12} \mathrm{O}: \mathrm{C}, 83.69 ; \mathrm{H}, 7.02$. Found: C, 83.49; H, 7.05.

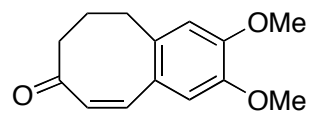

2,3-Dimethoxy-9,10-dihydrobenzocycloocten-7(8H)-one (5d). A mixture of 1d (69.7 mg, $0.30 \mathrm{mmol})$ and $\mathrm{Yb}(\mathrm{OTf})_{3}(37.2 \mathrm{mg}, 0.060 \mathrm{mmol})$ in $\mathrm{PhCl}(6.0 \mathrm{~mL})$ was heated under reflux for $2 \mathrm{~h}$. The reaction mixture was passed through a plug of Florisil $^{\circledR}$ (AcOEt). Purification by preparative thin-layer chromatography of silica gel (hexane:AcOEt = 5:2) afforded 5d (58.9 mg, 85\%): ${ }^{1} \mathrm{H}$ NMR (300 MHz) $\delta 2.09$ (quint, $\left.J=6.7 \mathrm{~Hz}, 2 \mathrm{H}\right), 2.37(\mathrm{t}, J=$ $6.7 \mathrm{~Hz}, 2 \mathrm{H}), 2.71$ (t, $J=6.7 \mathrm{~Hz}, 2 \mathrm{H}), 3.89$ (s, 3H), $3.92(\mathrm{~s}, 3 \mathrm{H}), 6.08$ (d, J= $12.8 \mathrm{~Hz}, 1 \mathrm{H})$, $6.75(\mathrm{~d}, J=10.5 \mathrm{~Hz}, 2 \mathrm{H}), 7.02(\mathrm{~d}, J=12.8 \mathrm{~Hz}, 2 \mathrm{H}) ;{ }^{13} \mathrm{C} \mathrm{NMR} \delta 32.0,33.2,38.0,55.9,56.0$, 112.2, 113.2, 128.0, 130.5, 134.4, 141.0, 147.2, 150.0, 204.3; HRMS calcd for $\mathrm{C}_{14} \mathrm{H}_{16} \mathrm{O}_{3}$ $\left(\mathrm{M}^{+}\right)$232.1099, found 232.1096.

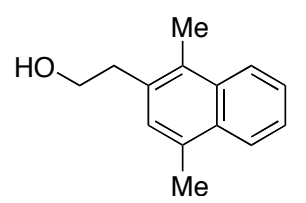

2-(1,4-Dimethylnaphthalen-2-yl)ethanol (2e-OH). ${ }^{8}$ A mixture of $\mathbf{1 e}(96.0 \mathrm{mg}, 0.48 \mathrm{mmol})$ and $\mathrm{Yb}(\mathrm{OTf})_{3}(59.5 \mathrm{mg}, 0.096 \mathrm{mmol})$ in $\mathrm{PhCl}(2.4 \mathrm{~mL})$ was heated under reflux for $3 \mathrm{~h}$. The reaction mixture was passed through a plug of Florisil ${ }^{\circledR}$ (AcOEt). After washing with $\mathrm{K}_{2} \mathrm{CO}_{3}$ aqueous solution, purification by preparative thin-layer chromatography of silica gel (hexane:AcOEt = 5:1) afforded 2e-OH $(50.3 \mathrm{mg}, 52 \%):{ }^{13} \mathrm{C}$ NMR $\delta 14.3,19.3,34.8,71.4$, $124.42,124.45,124.6,125.4,129.4,129.5,131.6,131.8,133.0,133.1$.

(8) [147595-50-4]. Sharma, P. K. Synth. Commun. 1993, 23, 389.9 


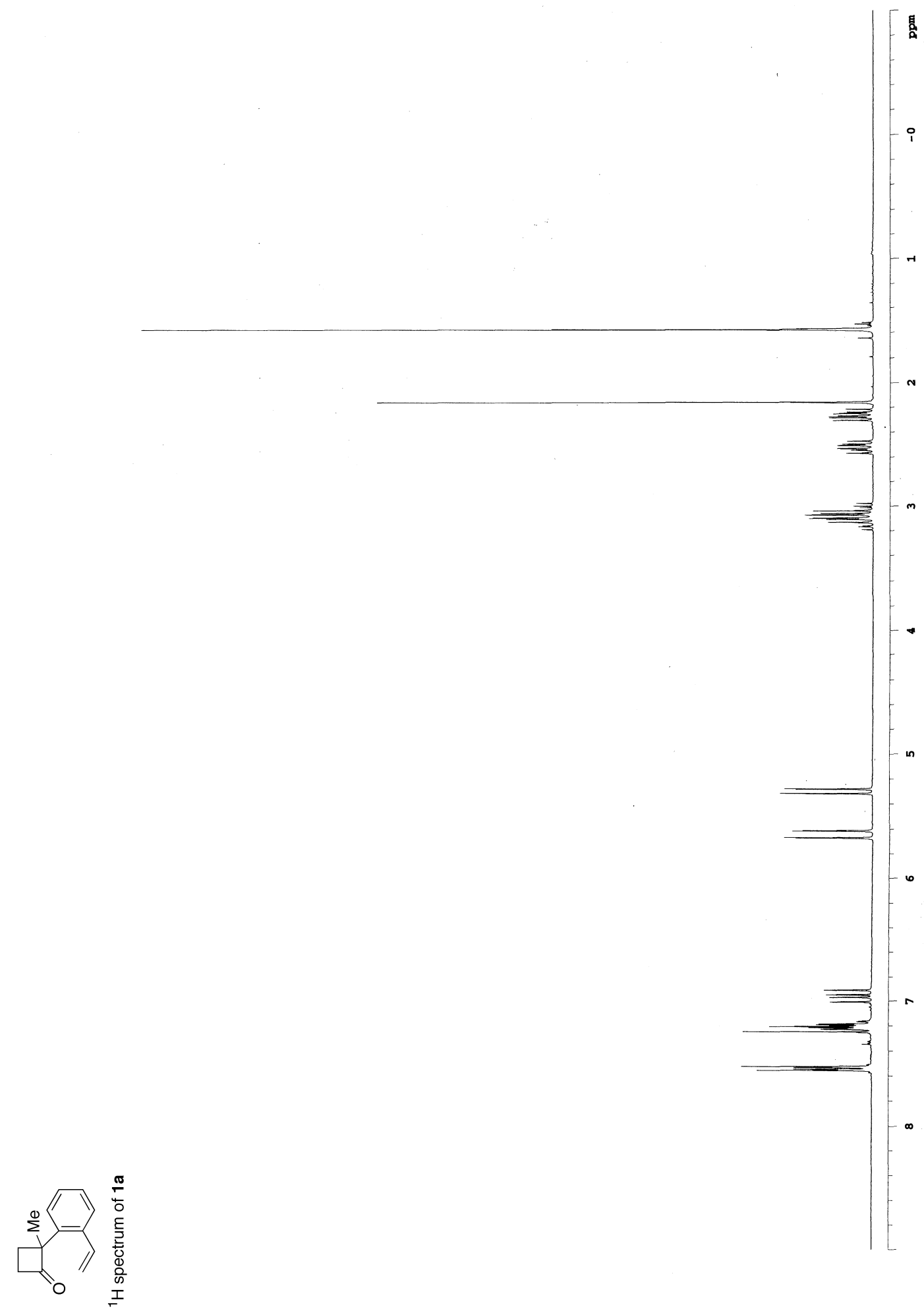




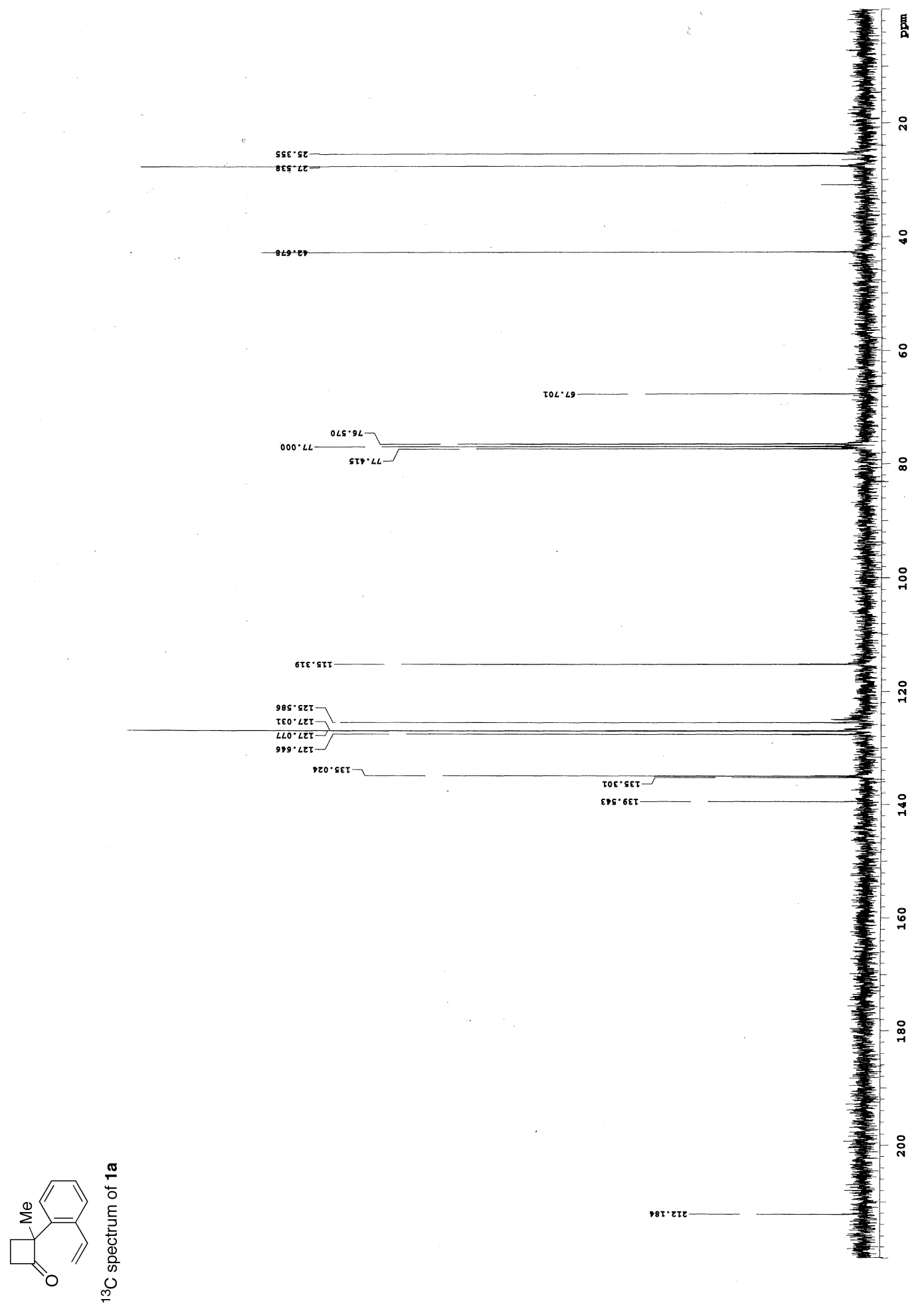




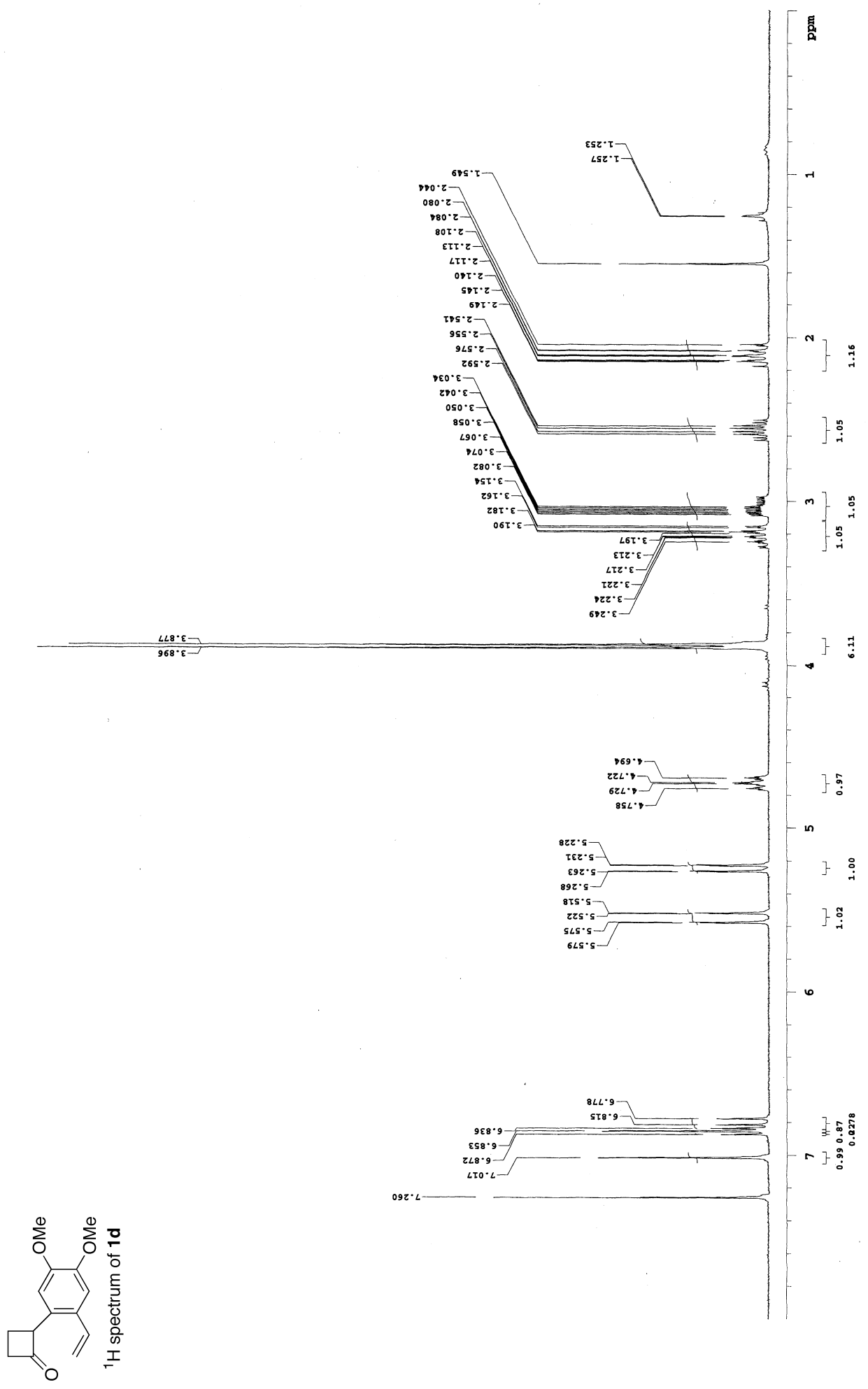




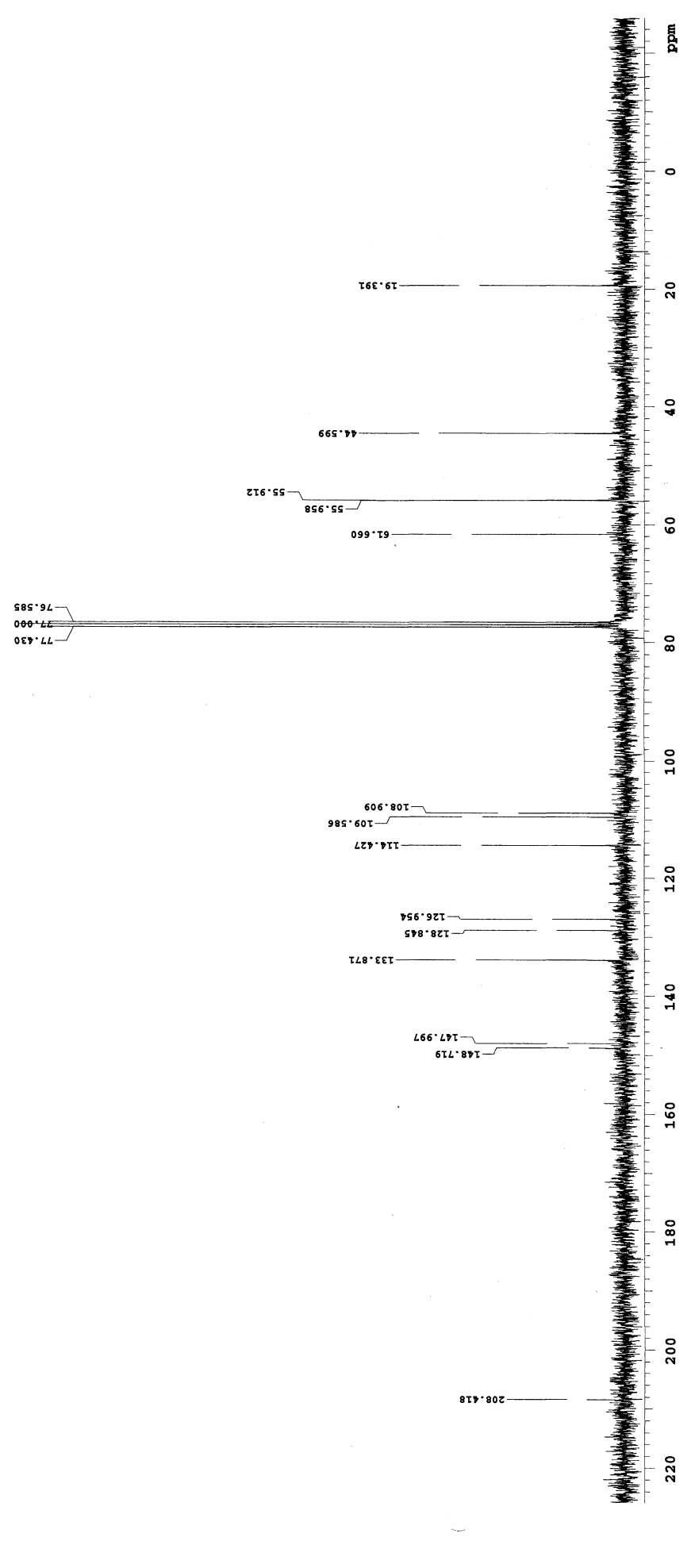




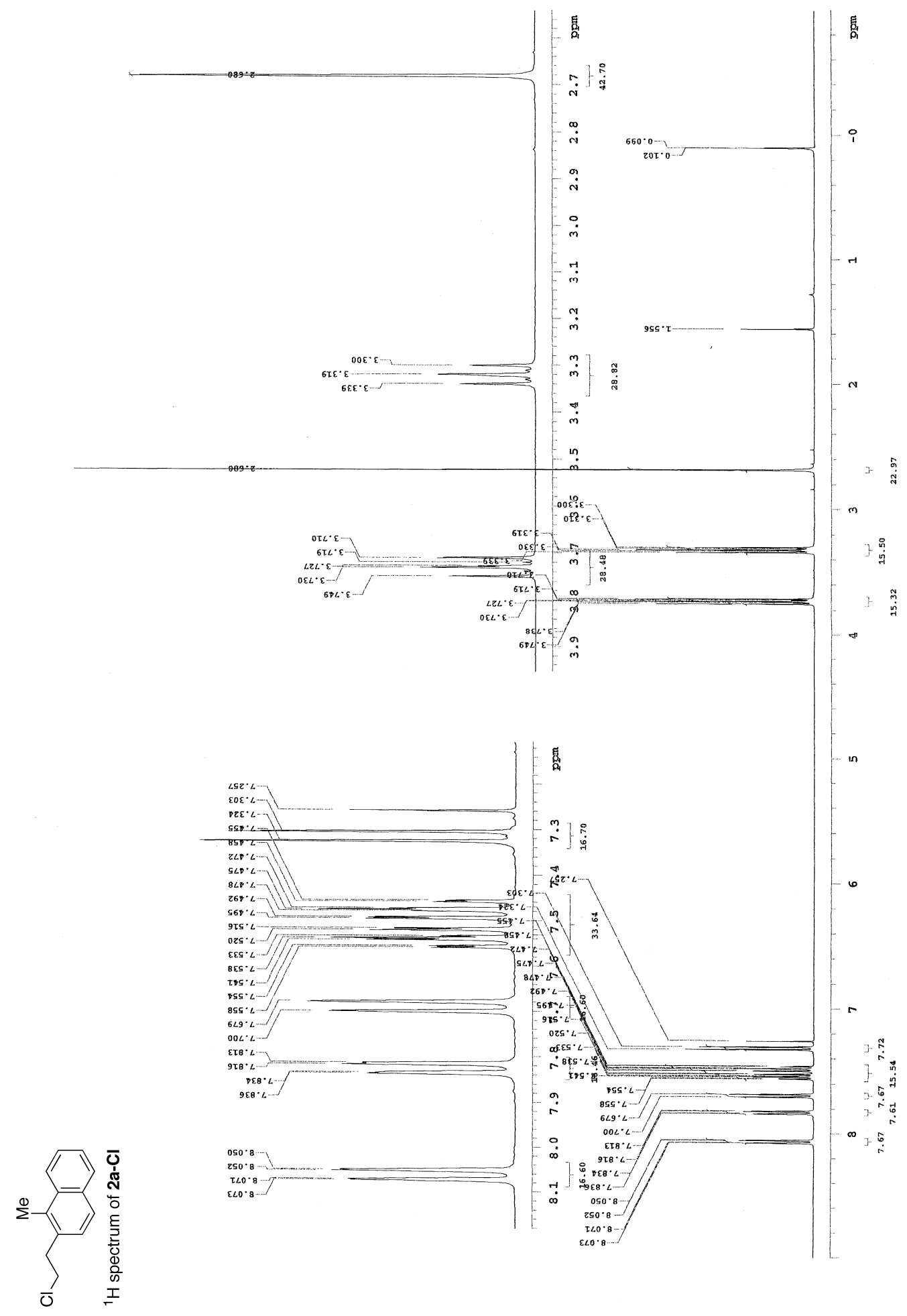




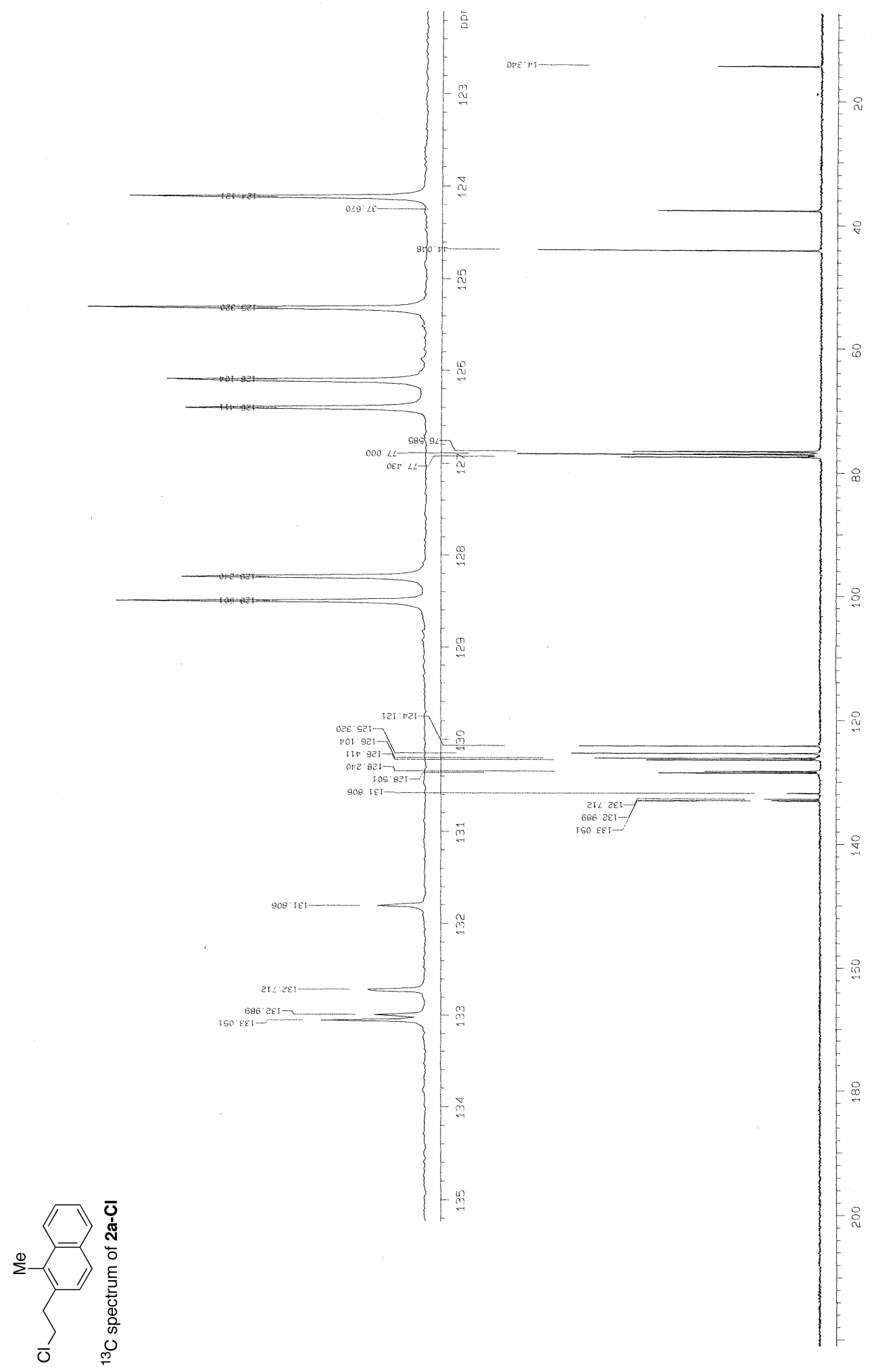




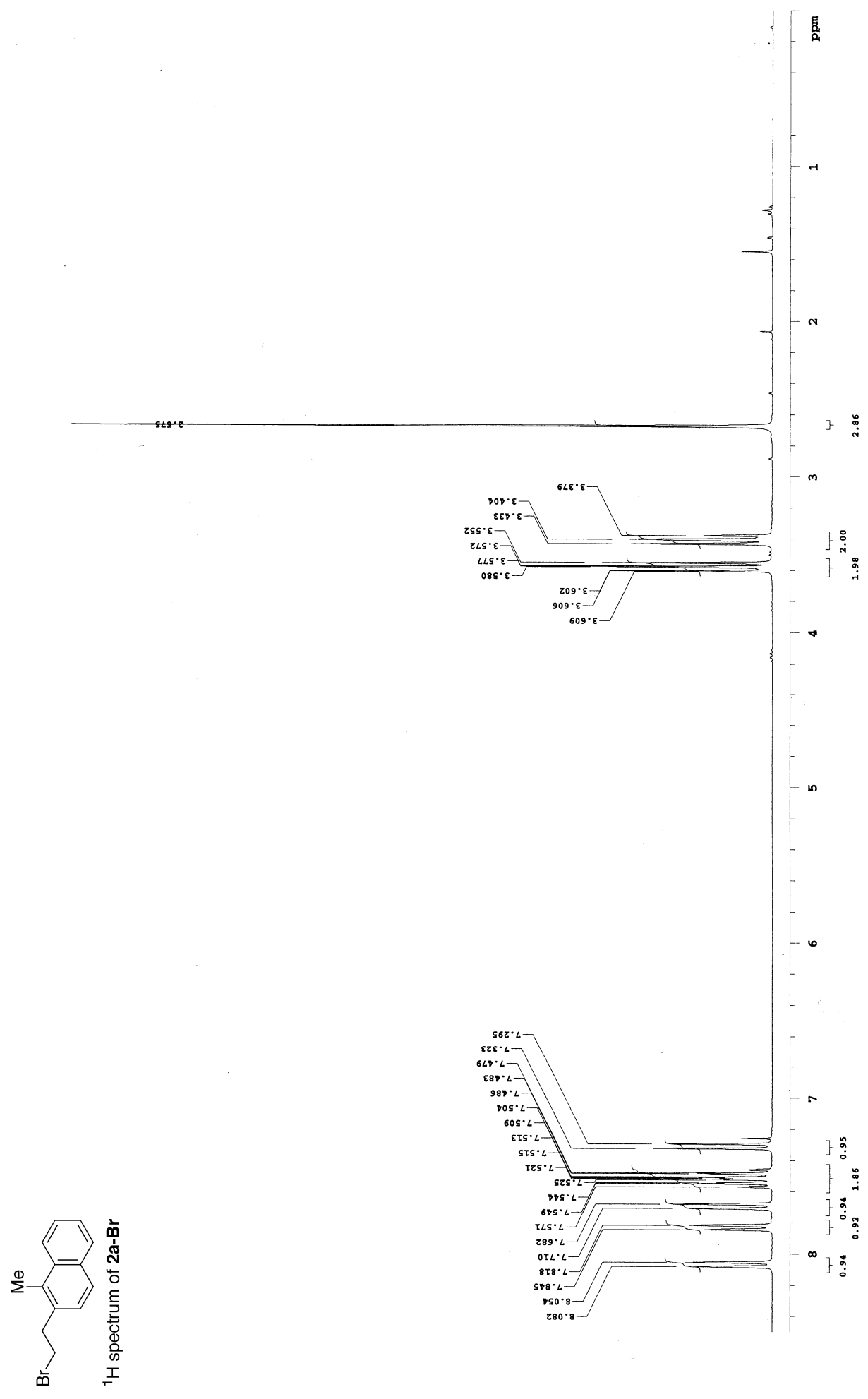




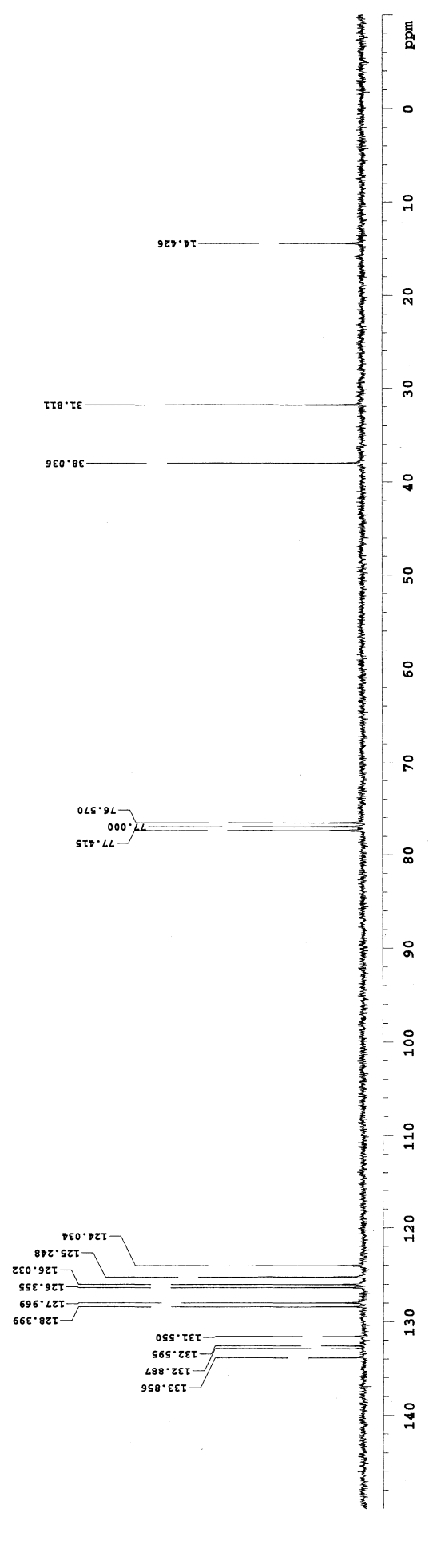




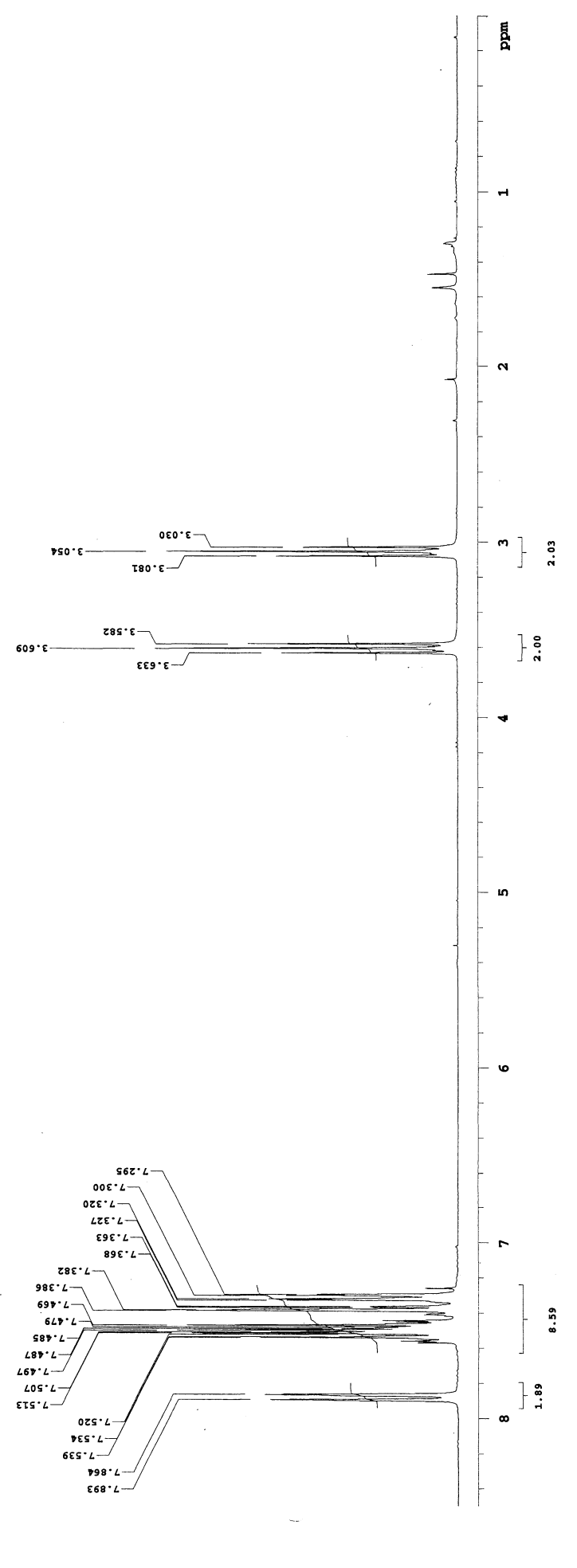




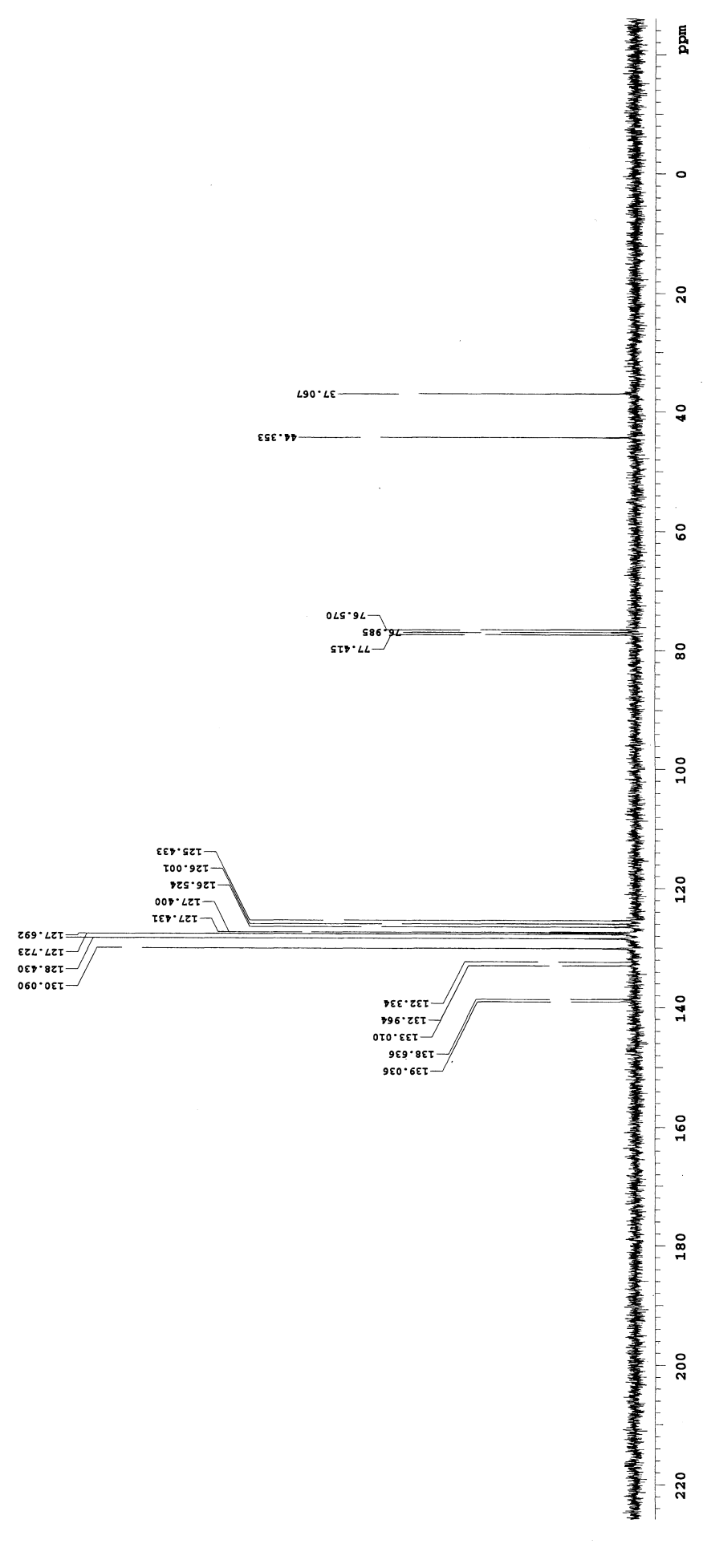




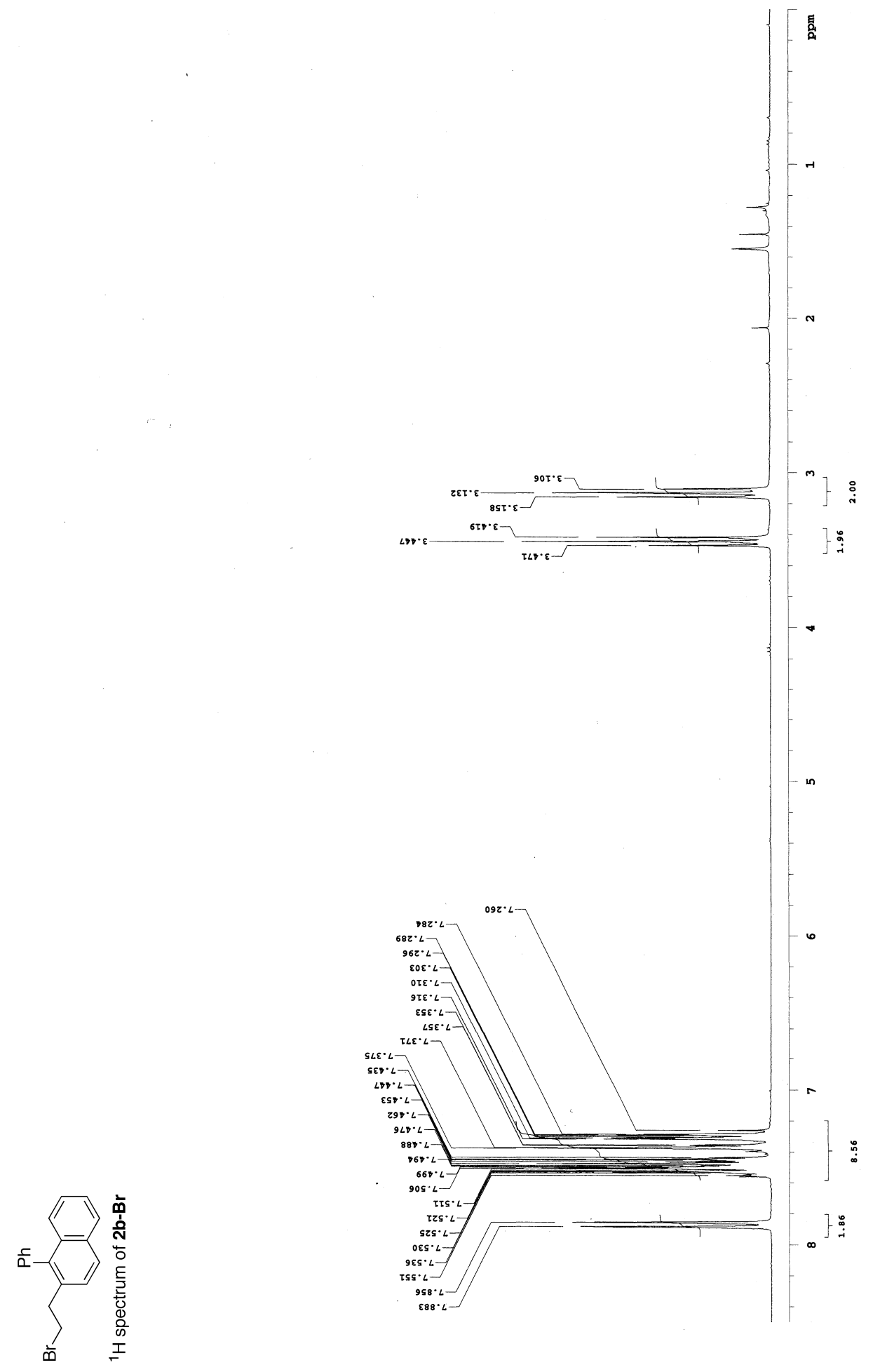



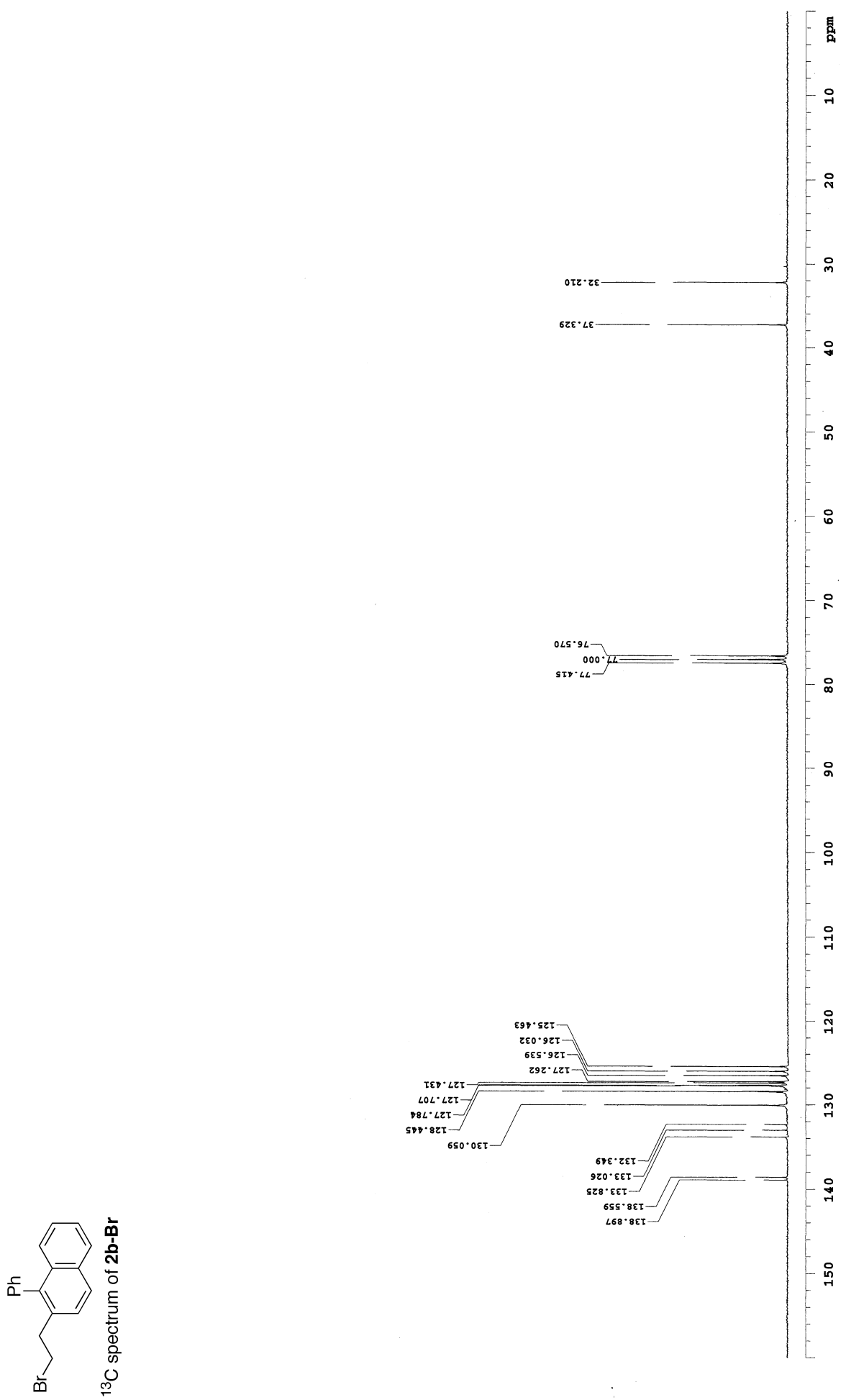


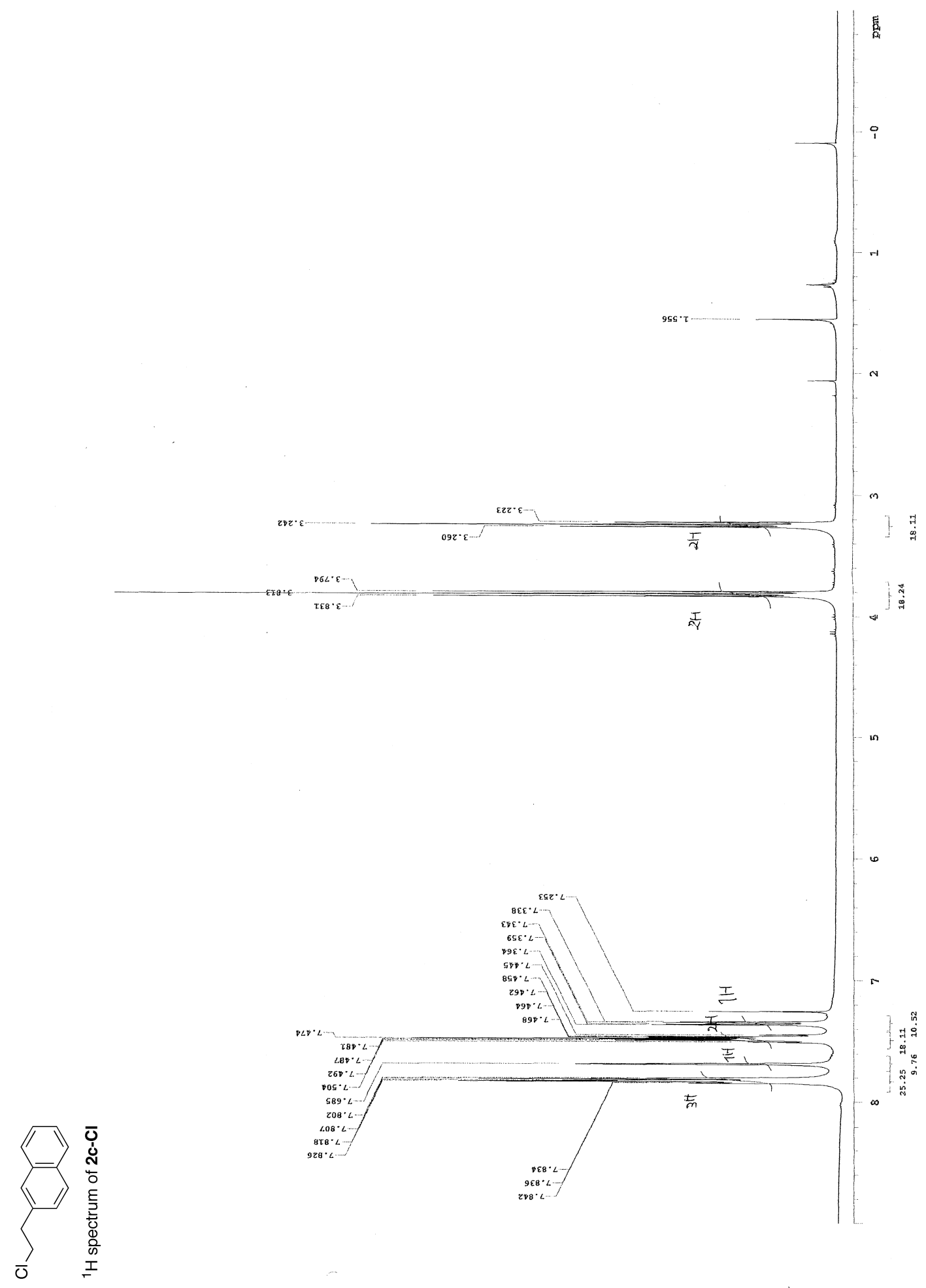




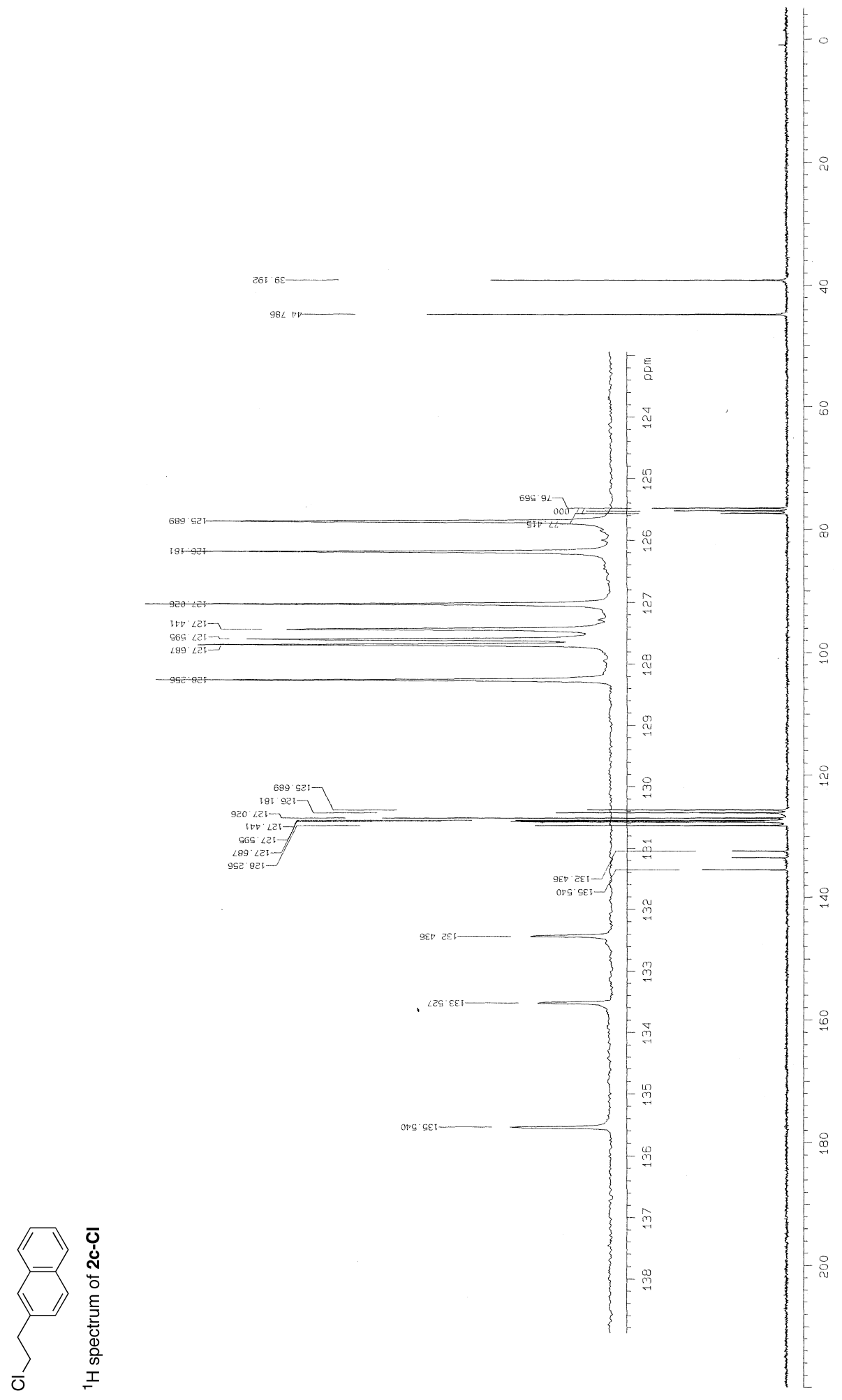




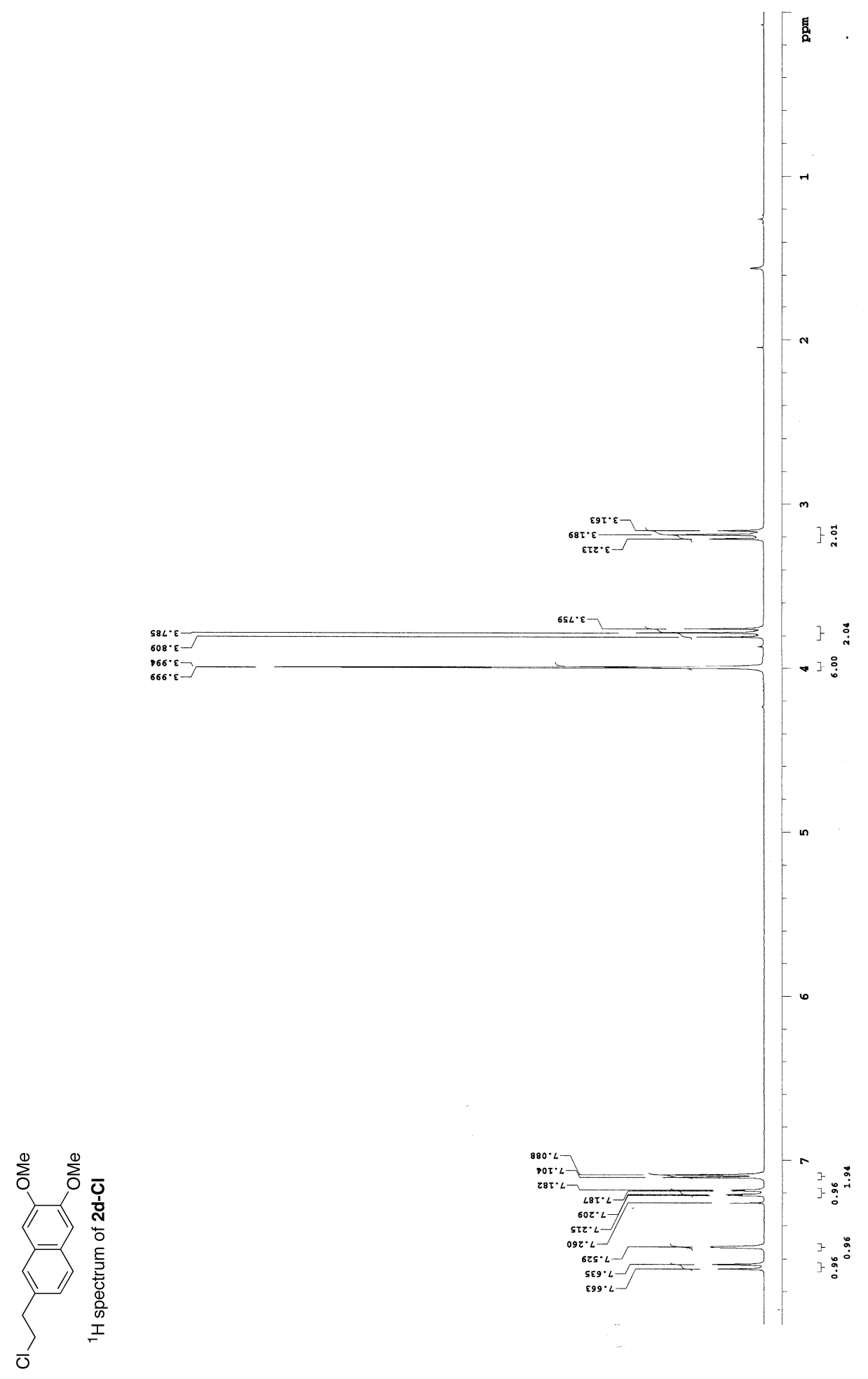



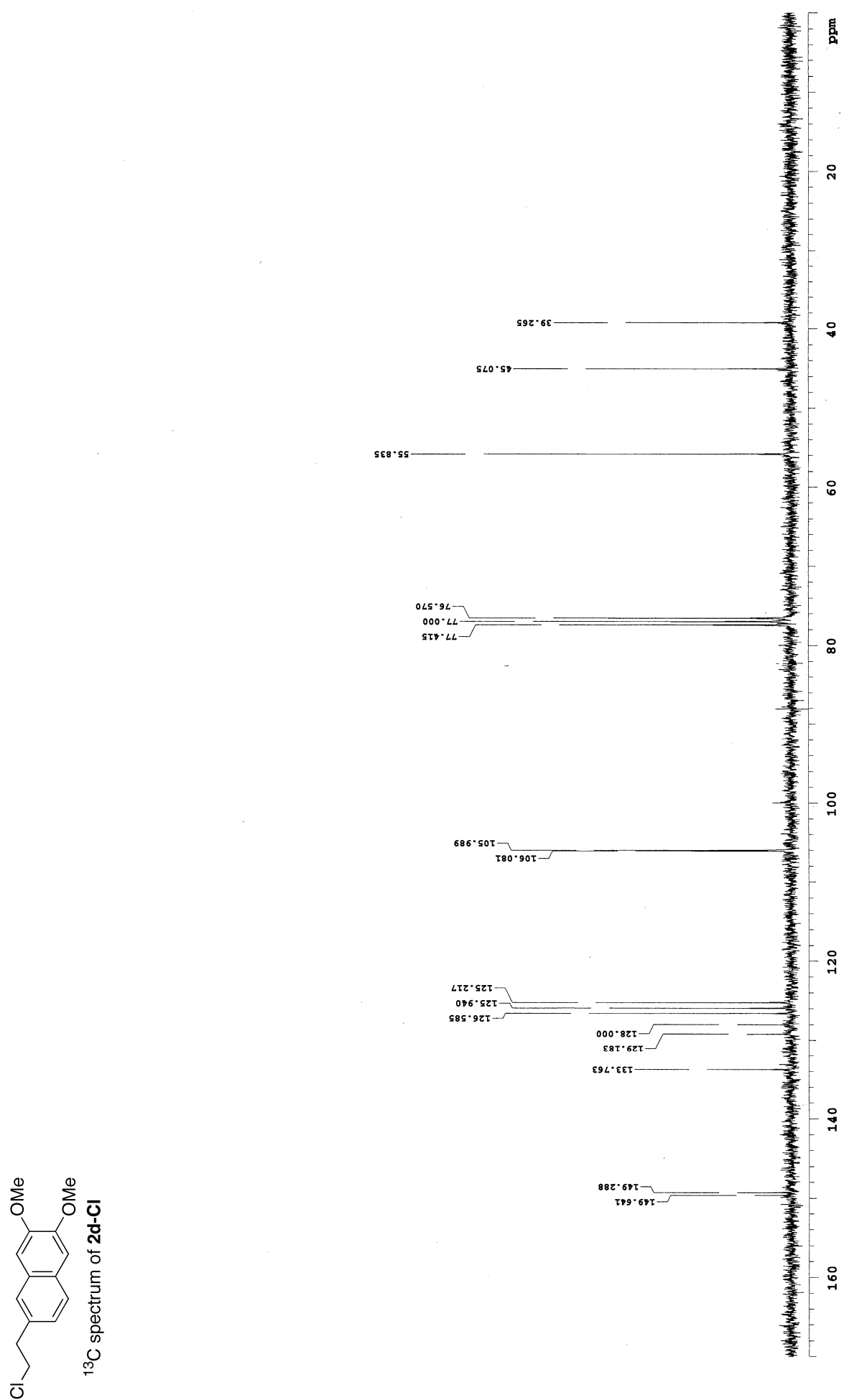


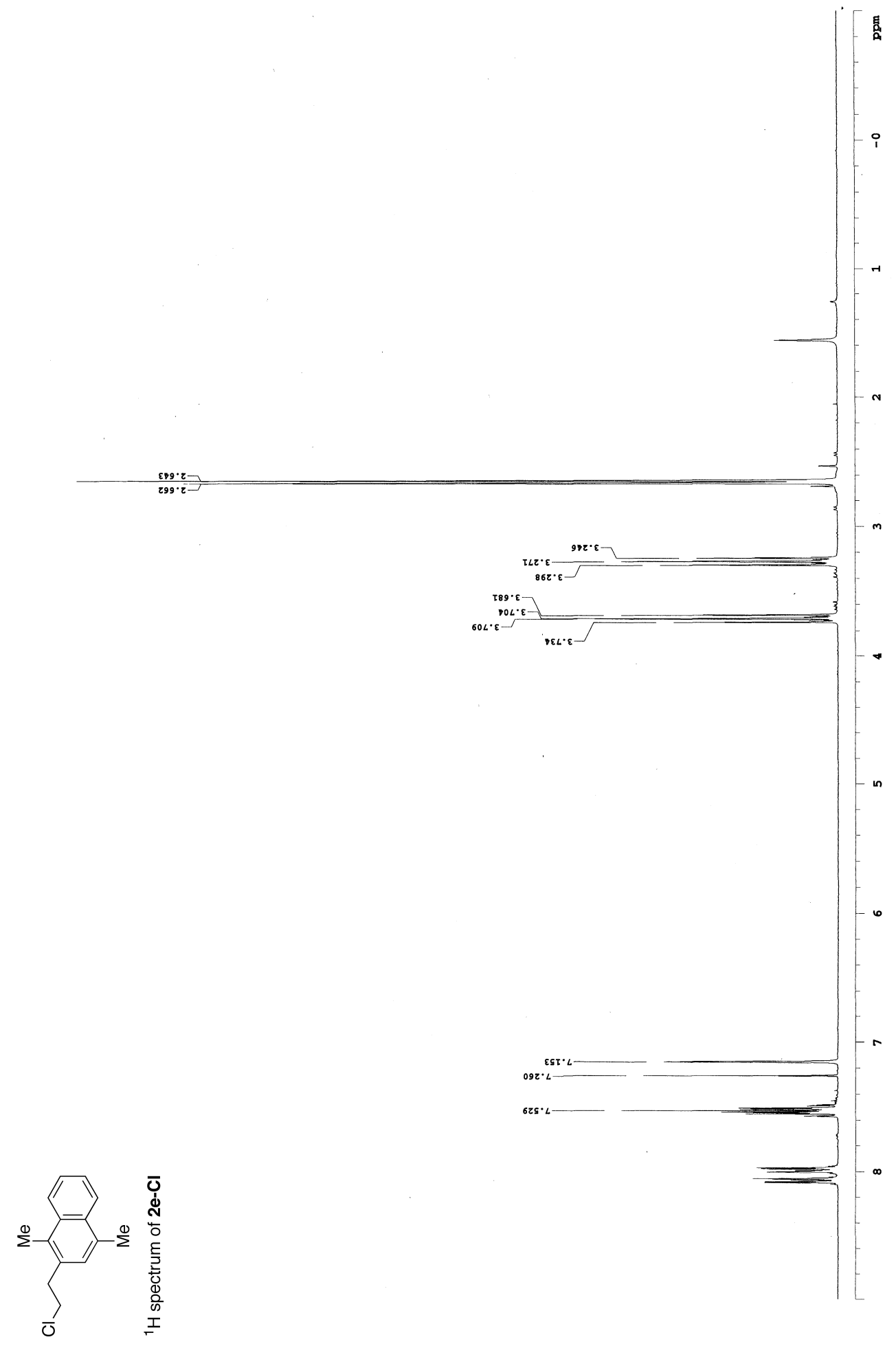




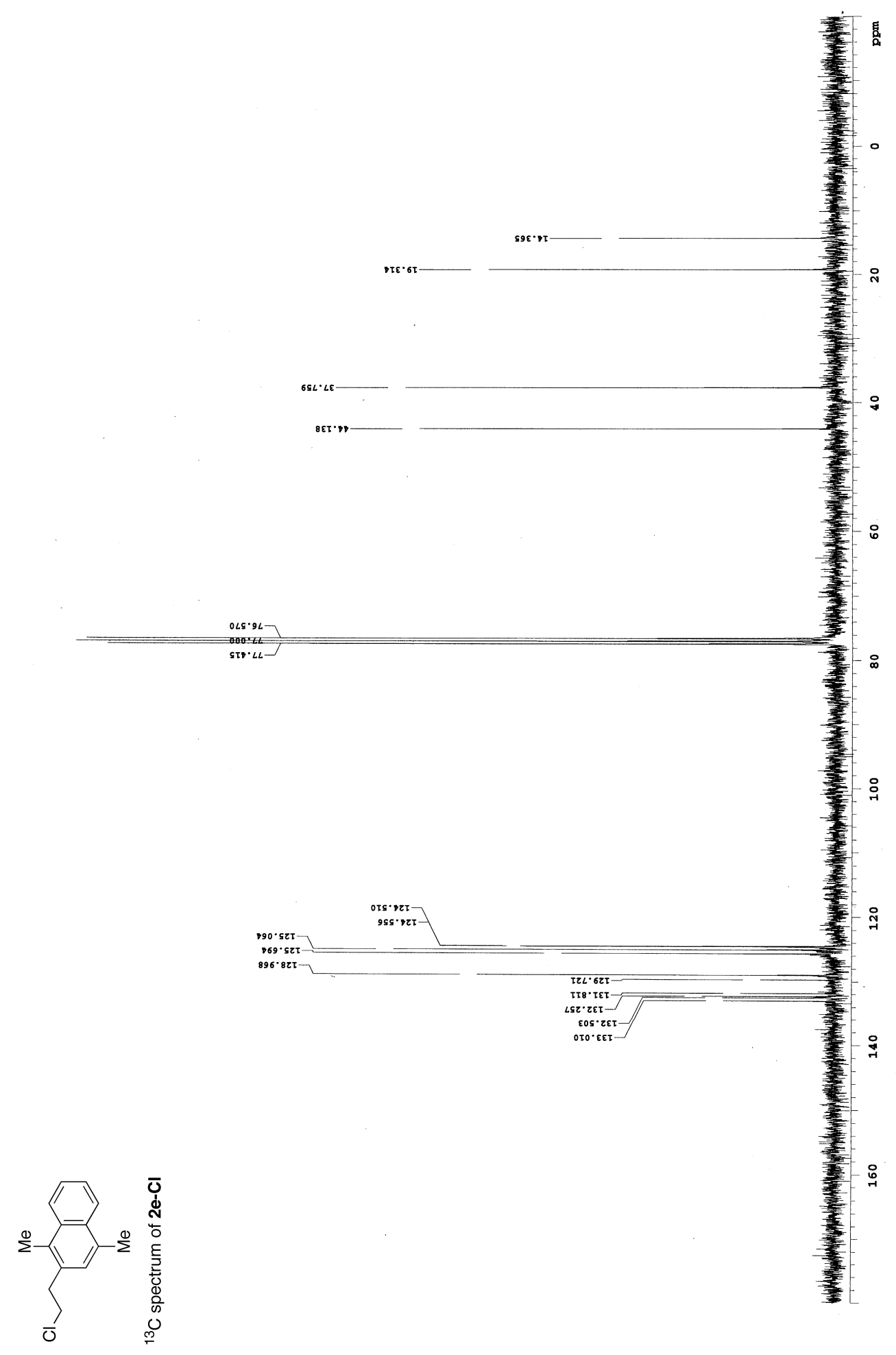




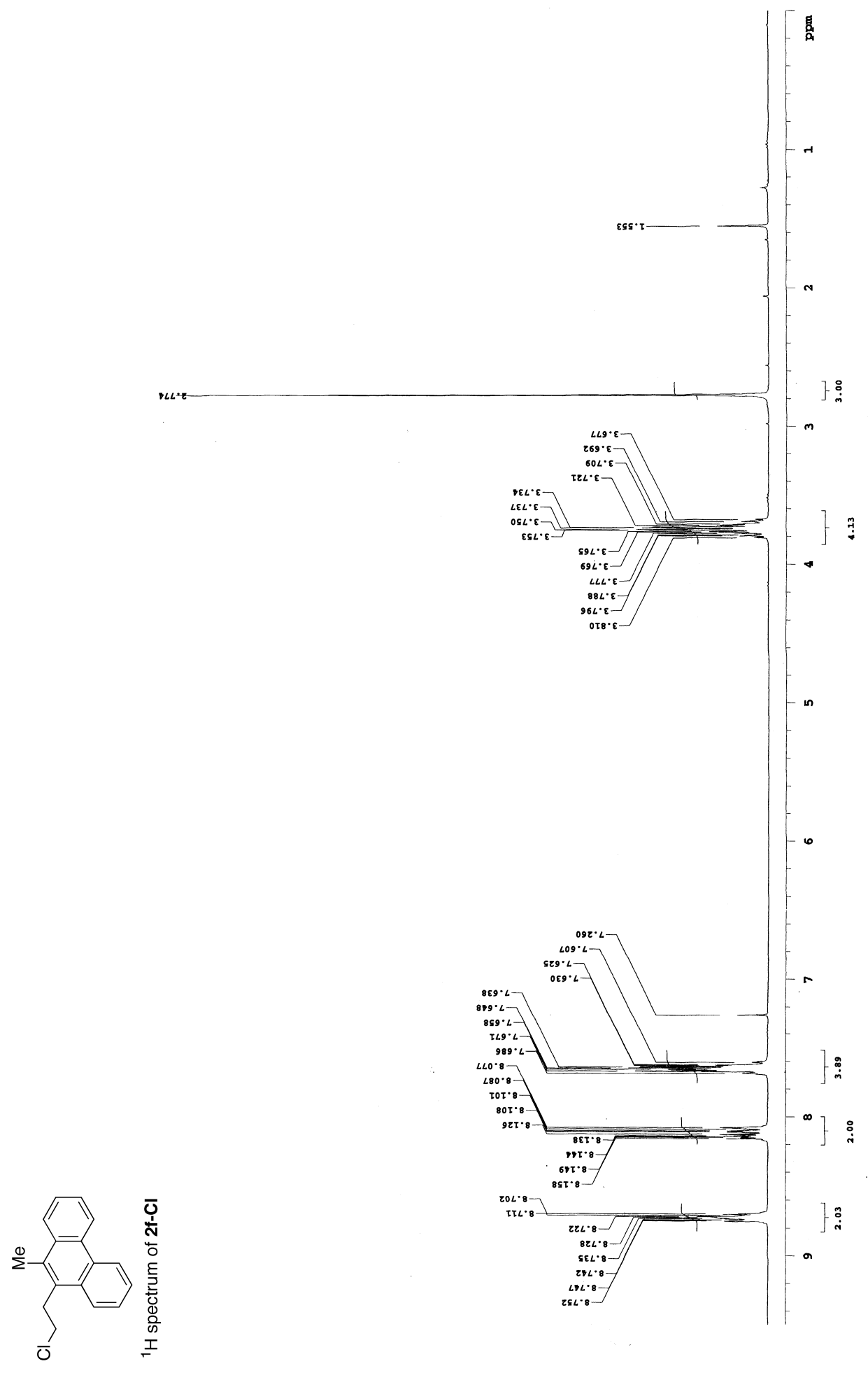




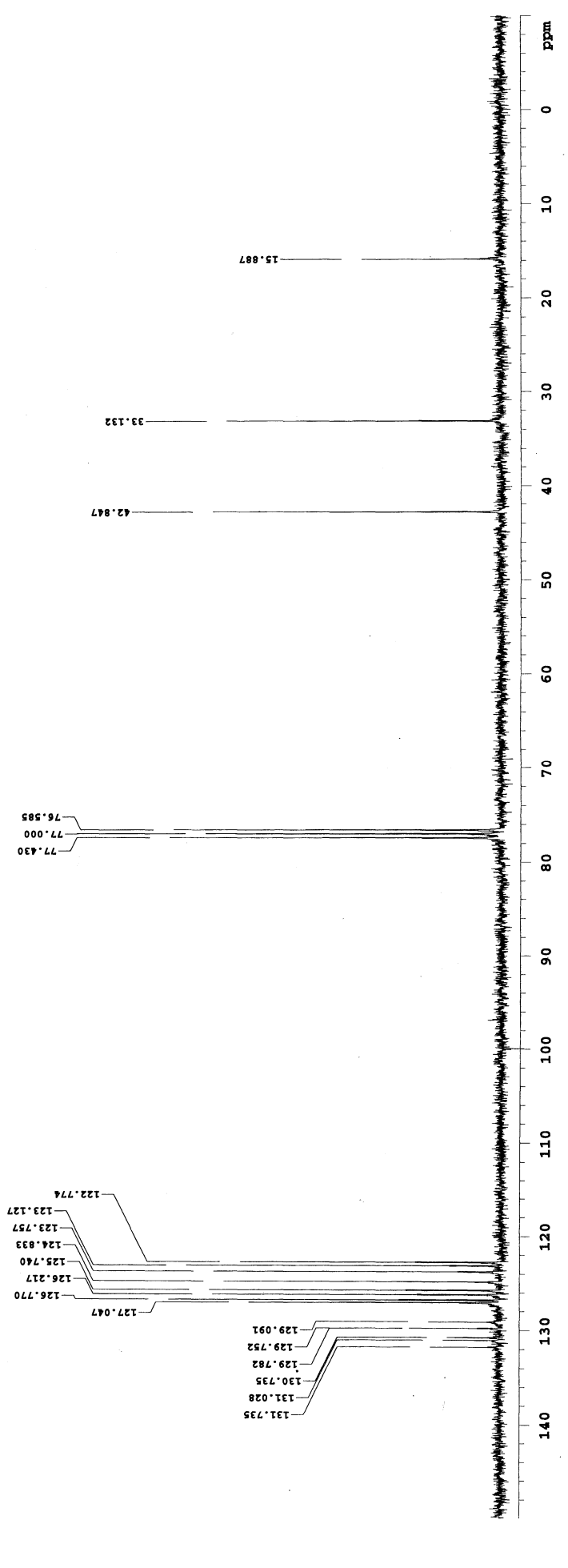




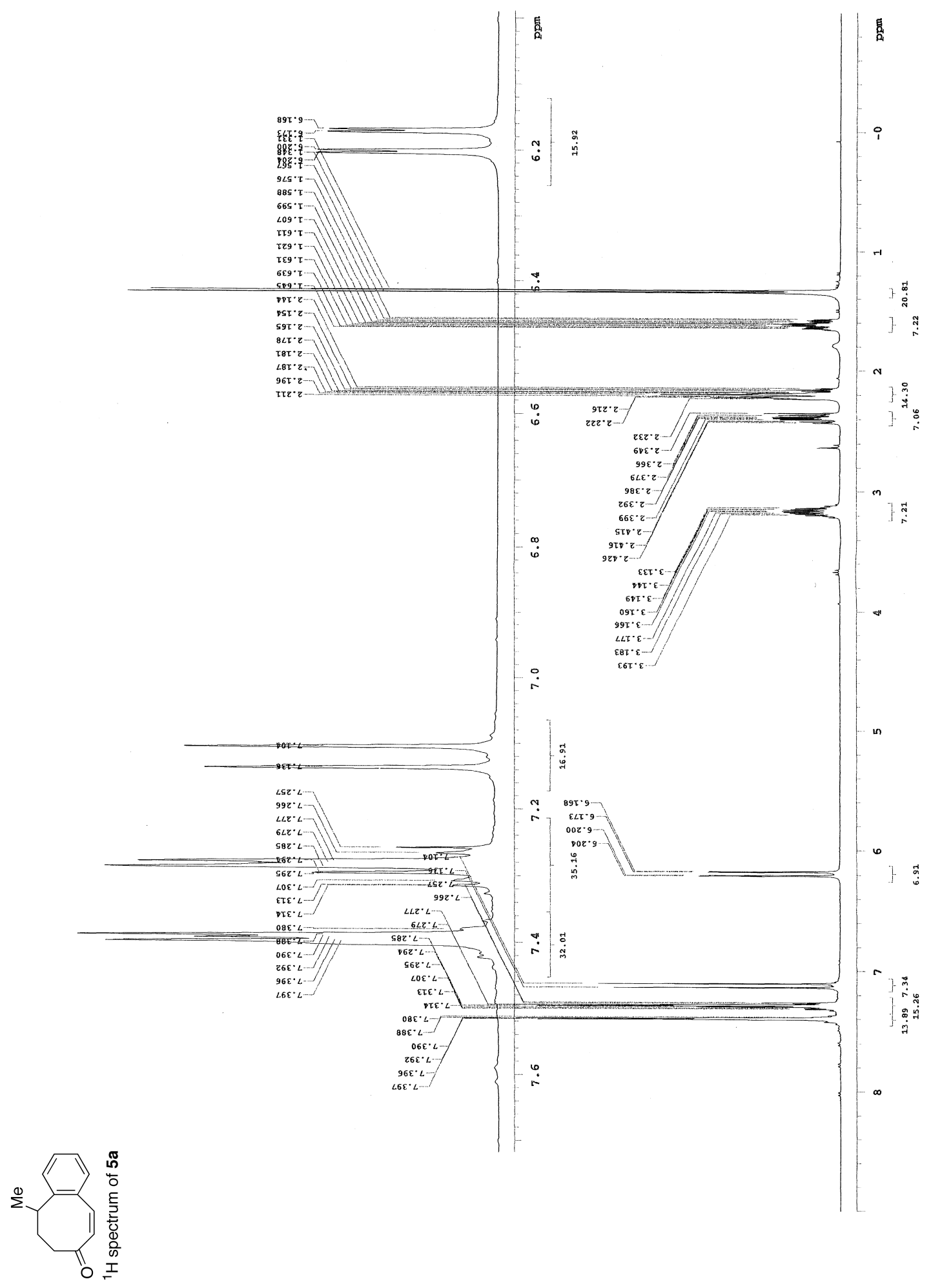




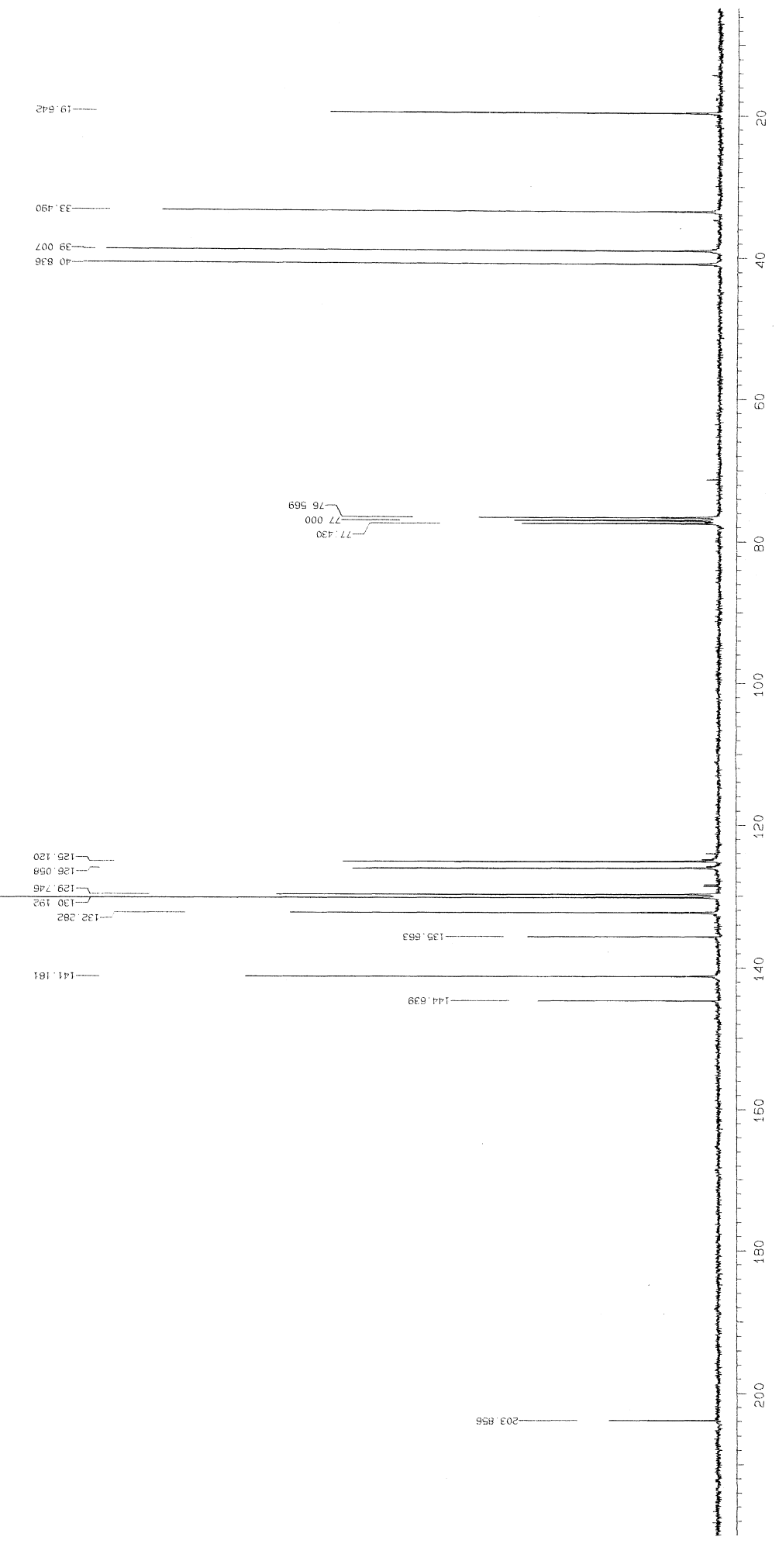




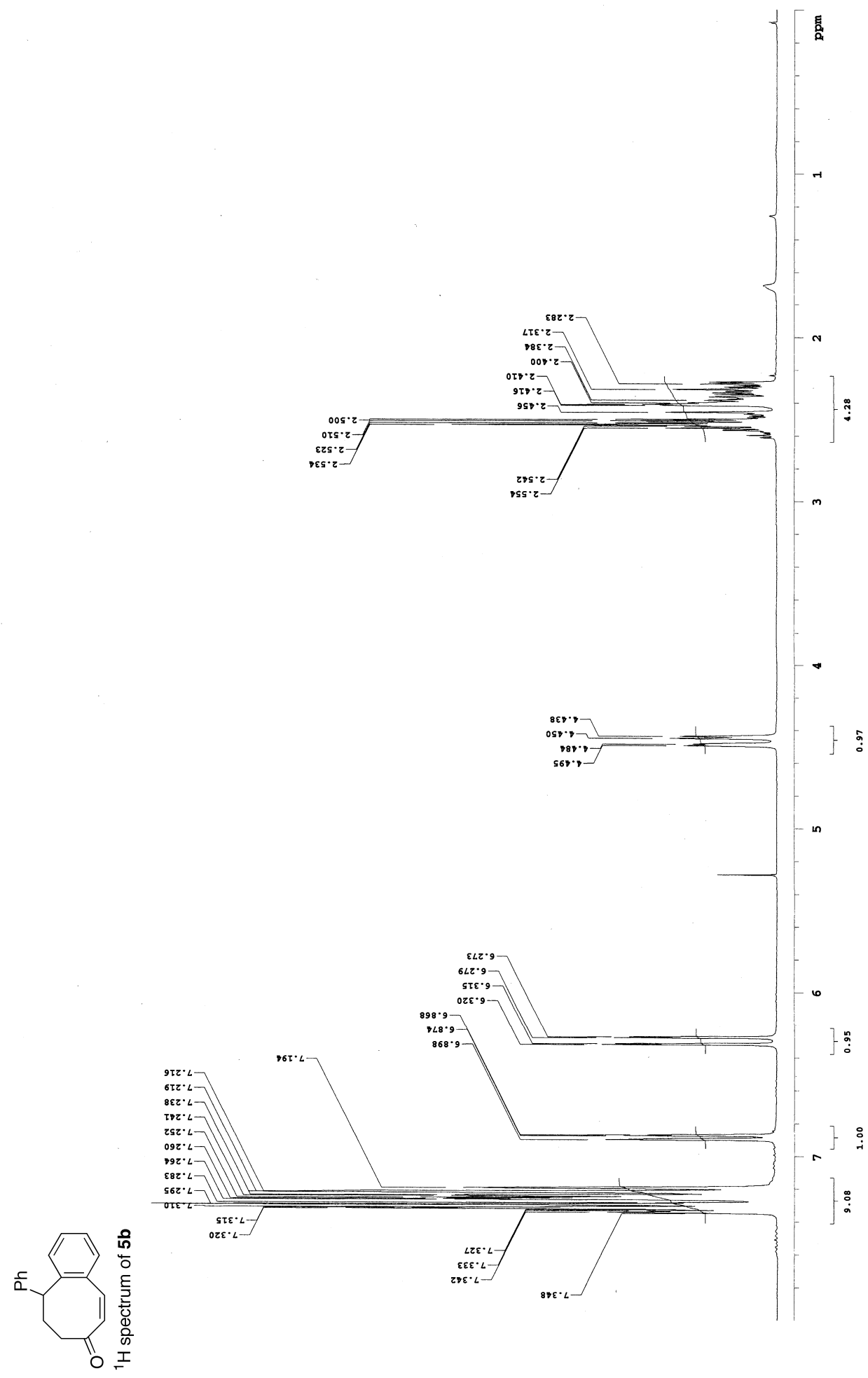




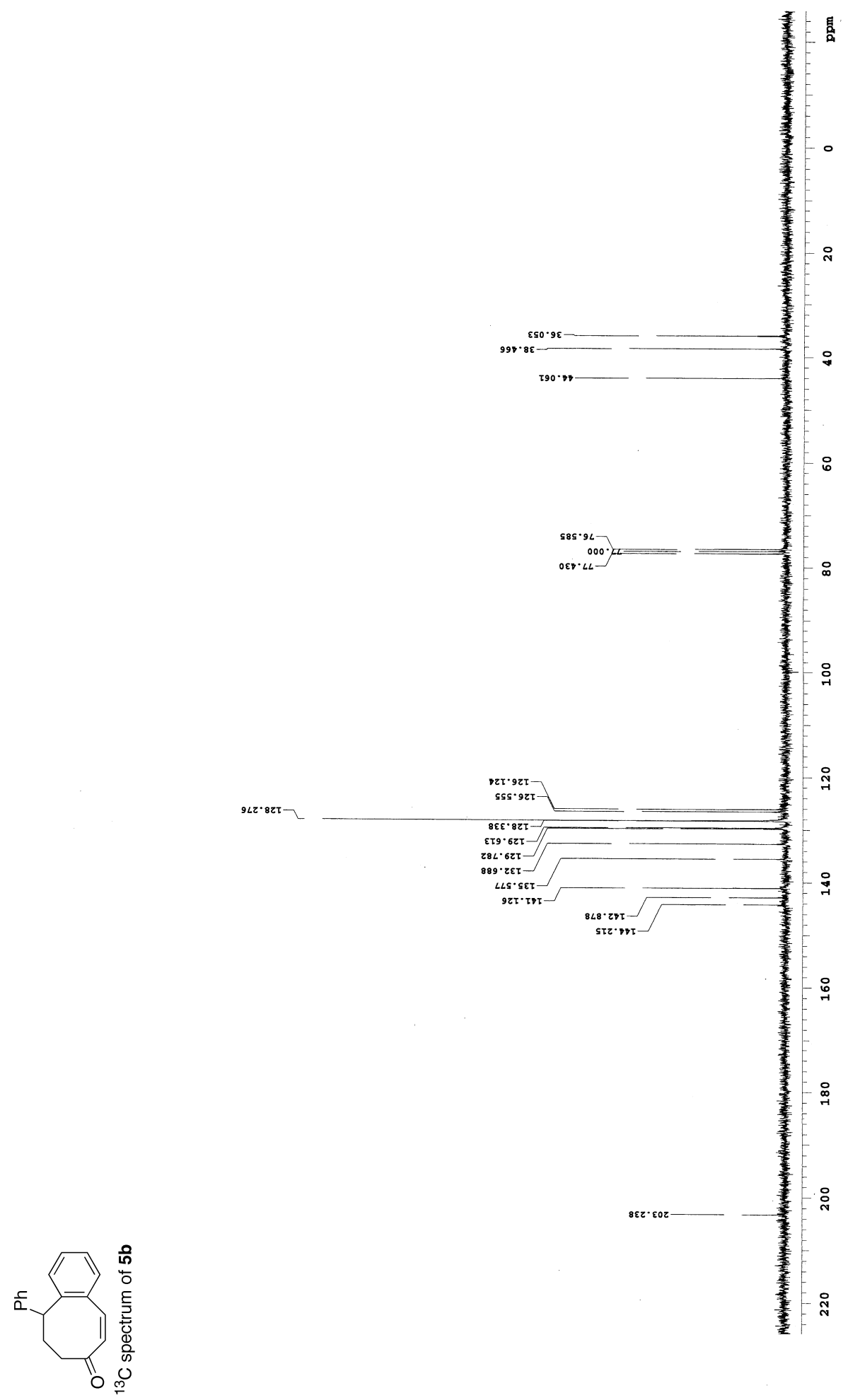




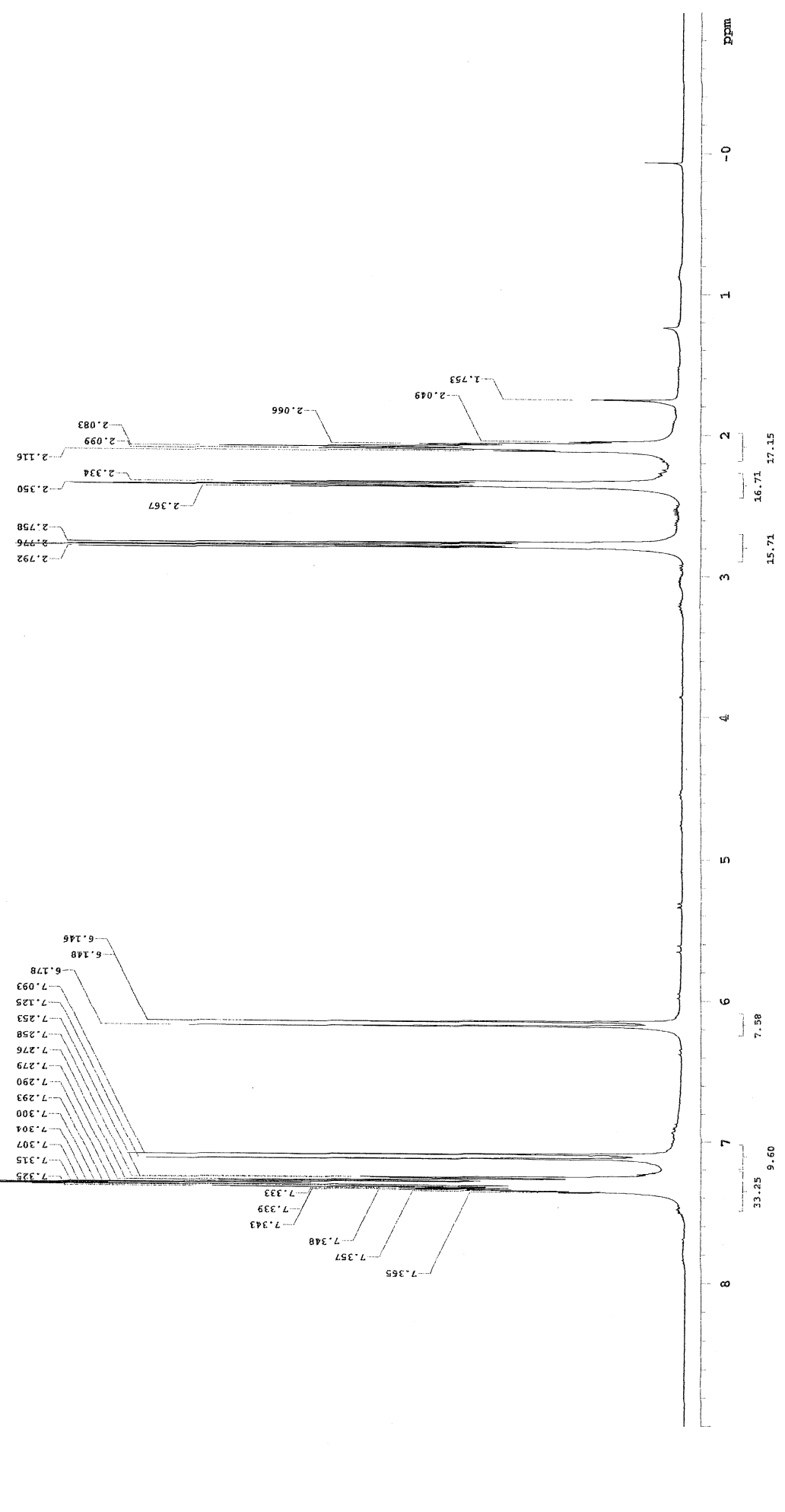




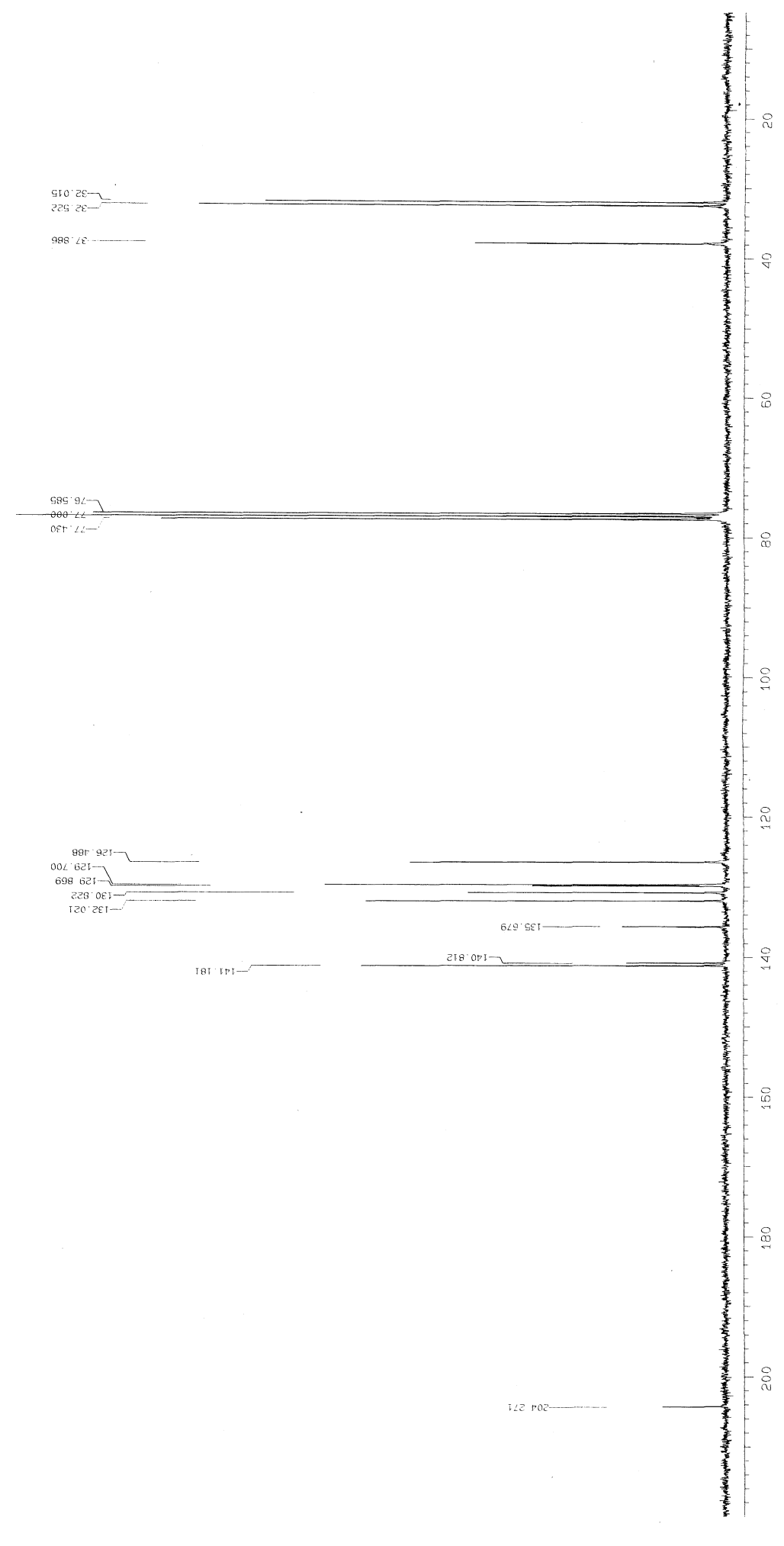




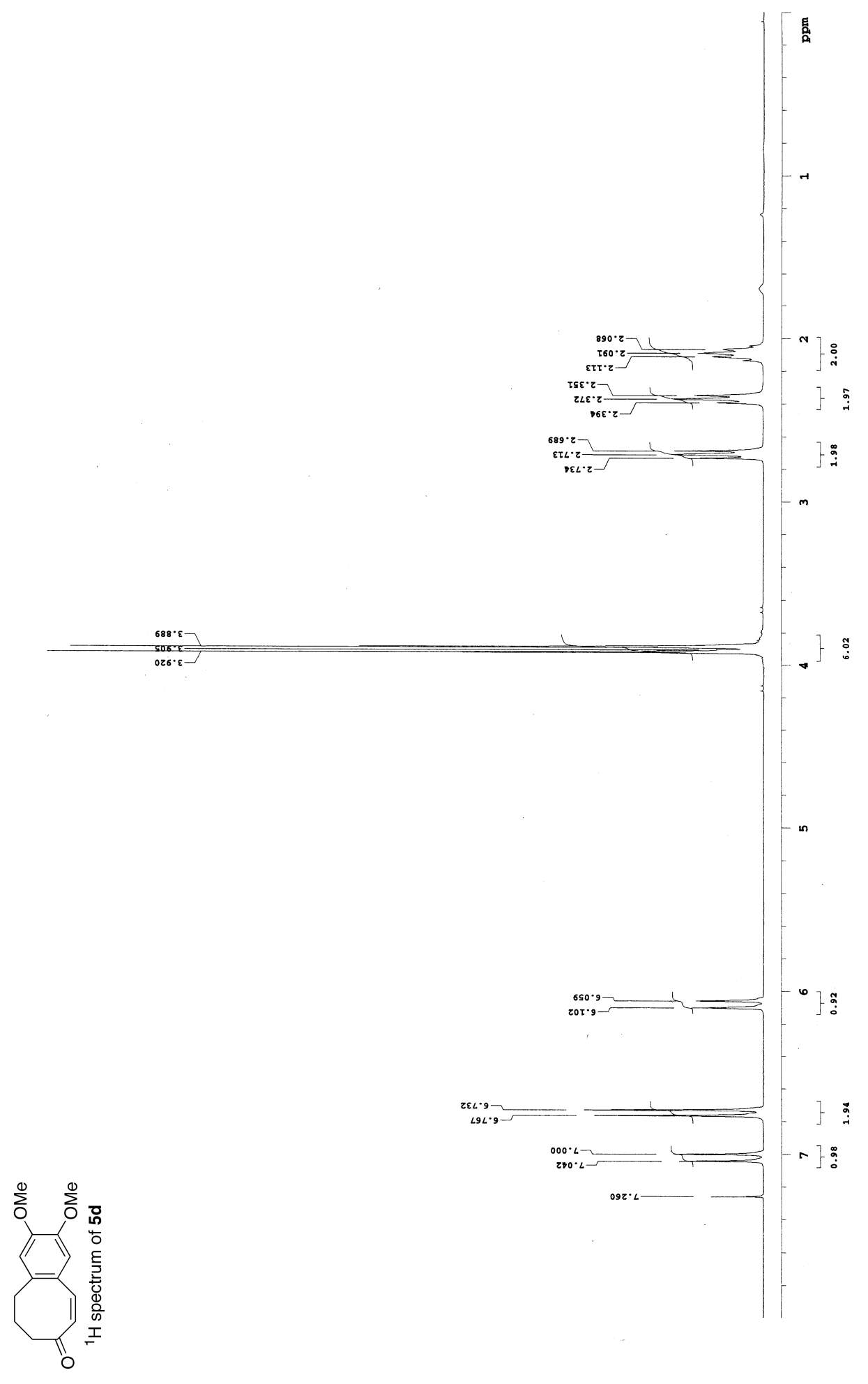




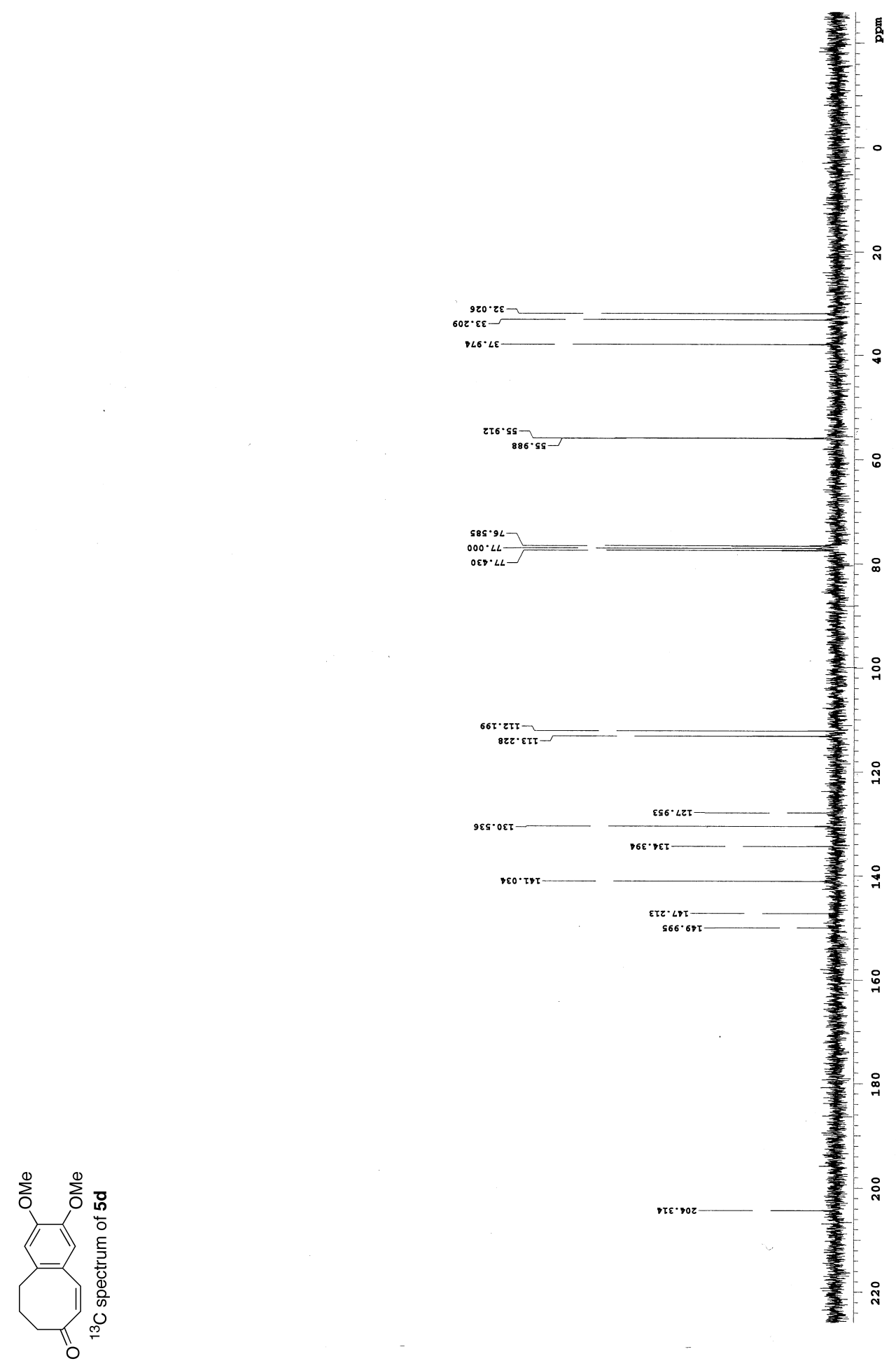




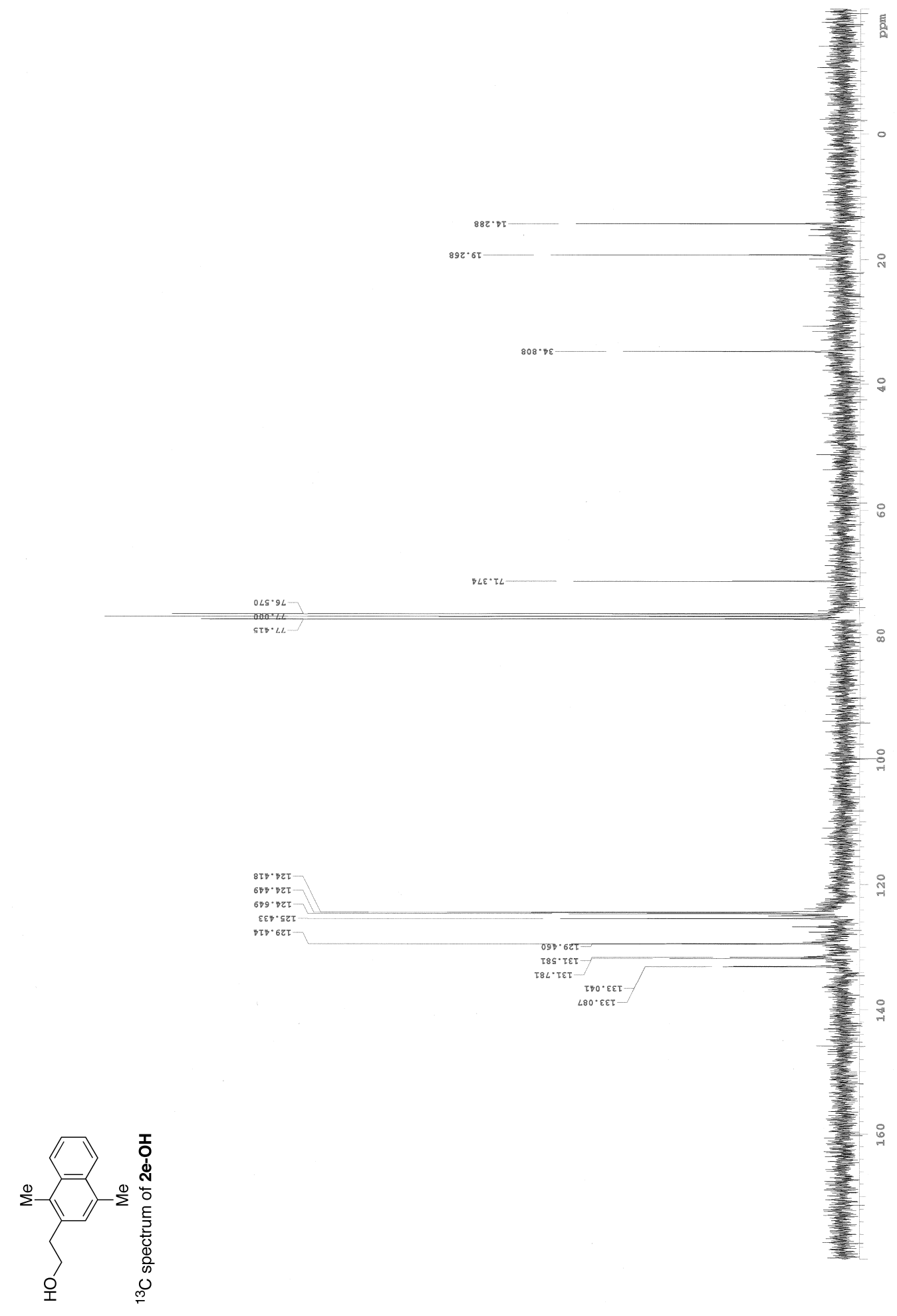

